\title{
Ferroelectric Behavior in Exfoliated 2D Aurivillius Oxide Flakes of Sub-Unit Cell Thickness
}

\author{
Lynette Keeney ${ }^{1 *}$, Ronan J. Smith ${ }^{2}$, Meghdad Palizdar ${ }^{3}$, Michael Schmidt ${ }^{1}$, Andrew J. Bell ${ }^{3}$, \\ Jonathan N. Coleman ${ }^{2}$ and Roger W. Whatmore ${ }^{4,5}$.
}

1. Tyndall National Institute, University College Cork, 'Lee Maltings', Dyke Parade, Cork, Ireland.

2. School of Physics and Centre for Research on Adaptive Nanostructures and Nanodevices, Trinity College, Dublin, D2 Dublin, Ireland.

3. Institute for Materials Research, University of Leeds, Leeds LS2 9JT, United Kingdom.

4. Department of Chemistry, University College Cork, Cork, Ireland.

5. Department of Materials, Faculty of Engineering, Imperial College London, London, SW7 2AZ, United Kingdom.

\begin{abstract}
2D materials, which are self-supporting in large areas but are only a few atoms thick, are exciting considerable interest for potential applications as diverse as electronics, molecular filtration and structural materials. Ferroelectrics have many useful applications stemming from their electrically-switchable dielectric spontaneous polarizations, including in piezoelectric devices, nonvolatile memories and pyroelectric IR sensors. While 2D oxide materials are known, they have not yet received the same attention as materials such as graphene. Here we study the ferroelectricity in ultrasonically-exfoliated flakes of the layered Aurivillius oxide $\mathrm{Bi}_{5} \mathrm{Ti}_{3} \mathrm{Fe}_{0.5} \mathrm{Co}_{0.5} \mathrm{O}_{15}$ with a range of thicknesses. We show, for the first time, that ferroelectricity can exist in flakes with thicknesses of only $2.4 \mathrm{~nm}$, which is about one-half of the normal crystal unit cell. These flakes have relatively large areas (linear dimensions many times the film thickness), thus classifying them as 2D materials. Piezoresponse force microscopy (PFM) has been used to show that these very thin flakes both
\end{abstract}


exhibit piezoelectric effects and that the ferroelectric polarization can be reversibly switched. A new model is presented that permits the accurate modelling of the field-on and field-off PFM timedomain and hysteresis loop responses from a ferroelectric during switching in the presence of charge injection, storage and decay through a Schottky barrier at the electrode-oxide interface. This allows the extraction of parameters such as the coercive fields $\left(E_{c}\right)$, electrostrictive coefficients $\left(Q_{11}\right)$ and spontaneous polarizations $\left(P_{S}\right)$ for the flakes. It is found that the values of $P_{S}$ are around $0.04( \pm 0.02) \mathrm{Cm}^{-2}$, and $Q_{11}=2( \pm 0.1) \times 10^{-2} \mathrm{~m}^{4} \mathrm{C}^{-2}$. These are in good agreement with other ferroelectric Aurivillius oxides. $E_{c}$ scales with thickness $(t)$, closely following the semi-empirical scaling law expected for ferroelectric materials, vis: $E_{C} \propto t^{-\frac{2}{3}}$. We believe this constitutes the first evidence for ferroelectricity in a 2D oxide material, and it offers the prospect of new devices that might use the useful properties associated with the switchable ferroelectric spontaneous polarization in a 2D materials format. 


\section{Introduction}

2D materials, such as graphene, have received enormous attention over the last 10 years for their huge potential range of applications, ranging from electronics and photonics to structural materials, as has been comprehensively described elsewhere. ${ }^{[1]}$ The definition of a $2 \mathrm{D}$ material as being one of large area but only one atom thick (e.g. graphene) has been relaxed to include materials which can exist in large area, but a few atoms and typically ca 1 unit cell or less in thickness ${ }^{[1 \mathrm{a}]}$ and being self-supporting (without the necessity for a substrate). As a consequence, many 2D materials apart from graphene are being studied, such as the $\mathrm{MoS}_{2}$ family. Many of these materials are semiconductors. A variety of manufacturing techniques exist ${ }^{[1 \mathrm{~b}]}$, including mechanical exfoliation and direct growth. Liquid exfoliation (high shear \& ultrasonic) methods offer the prospect for the low-cost production of large volumes of flakes in suspension that can subsequently be used in a variety of ways. ${ }^{[2]} 2 \mathrm{D}$ functional oxides also exist and examples include simple transition metal oxides such as titania ${ }^{[3]}$ and perovskite ${ }^{[4]} \mathrm{La}_{0.95} \mathrm{Nb}_{2} \mathrm{O}_{7}$ nanosheets made by exfoliation, which have been studied for their potentially-high relative permittivities (45 to 100 ), ${ }^{[5]} \mathrm{MnO}_{2}$ flakes which have received attention for battery ${ }^{[6]}$ and supercapacitor applications ${ }^{[7]}$ and layered tantalates such as $\mathrm{H}_{1.81} \mathrm{Sr}_{0.81} \mathrm{Bi}_{0.19} \mathrm{Ta}_{2} \mathrm{O}_{7}$ being investigated for photocatalytic water splitting. ${ }^{\left[{ }^{8]}\right.}$ Recently, it has been shown that mechanically-exfoliated $2 \mathrm{D}$ flakes of the oxide superconductor $\mathrm{Bi}_{2} \mathrm{Sr}_{2} \mathrm{CaCu}_{2} \mathrm{O}_{8+\square}$ exhibit superconducting transition temperatures similar to the bulk when only one-half a unit cell in thickness. ${ }^{[9]}$

Ferroelectrics (FEs) offer a range of functional properties ${ }^{[10]}$ and are widely used in e.g. nonvolatile memories, ${ }^{[11]}$ piezoelectric RF Filters ${ }^{[12]}$ and pyroelectric infra-red detectors. ${ }^{[13]}$ FE switching has previously been reported in very thin films e.g. 7.5nm PVDF-TrFE copolymer films ${ }^{[14]}$ and films of $\mathrm{BaTiO}_{3}$ as thin as $3 \mathrm{~nm}$ (ca 8 perovskite unit cells or "blocks"). ${ }^{[15]}$ Recently, $\mathrm{FE}$ behavior has been reported in free-standing monolayer flakes of $\ln _{2} \mathrm{Se}_{3}{ }^{[16]}$ and $\mathrm{MoTe}_{2}{ }^{[17]}$ although their spontaneous polarizations $\left(P_{S}\right)$ have not been quantified and the difficulties of measuring $P_{S}$ in such narrow band- 
gap materials are widely acknowledged. ${ }^{[18]}$ The Aurivillius oxide layer-structure materials (APMs) ${ }^{[19]}$ _ general formula $\mathrm{Bi}_{2} \mathrm{O}_{2}\left(A_{m-1} B_{m} O_{3 m+1}\right)$ - consist of $m$ perovskite $\mathrm{ABO}_{3}$ blocks interspersed with fluorite $\mathrm{Bi}_{2} \mathrm{O}_{2}$ layers. Many APMs are $\mathrm{FE}$, e.g. $\mathrm{Bi}_{4} \mathrm{Ti}_{3} \mathrm{O}_{12} \cdot{ }^{[20]}$ Characteristically, they have high Curie temperatures $\left(>600^{\circ} \mathrm{C}\right)$, large in-plane $\mathrm{P}_{\mathrm{s}}\left(\mathrm{ca} 0.4 \mathrm{Cm}^{-2}\right)$ and in some cases small out-of-plane $\mathrm{Ps}_{\mathrm{s}}$. $\mathrm{SrBi}_{2} \mathrm{Ta}_{2} \mathrm{O}_{9}(m=2)$ and $\mathrm{Bi}_{3.25} \mathrm{La}_{0.75} \mathrm{Ti}_{3} \mathrm{O}_{12}(m=3)$ APMs have been developed for commercial use in FeRAM (FE random access memory) devices. ${ }^{[21]}$ APMs are structurally-flexible, as many different cations can be included in the perovskite layers, and $m$ can be varied from two up to nine. ${ }^{[22]}$ [23] APMs are amenable starting materials for exfoliation into 2D nano-sheets. ${ }^{[24]}$ However, to date there have been no studies reported of the FE properties of exfoliated APM nanoflakes. Here we report, for the first time, the study of ferroelectricity in thin exfoliated nanoflakes of the APM $\mathrm{Bi}_{5} \mathrm{Ti}_{3} \mathrm{Fe}_{0.5} \mathrm{CO}_{0.5} \mathrm{O}_{15}$ (B5TFCO) $(m=4)$ using piezoresponse force microscopy (PFM), with flake thicknesses ranging from ca $100 \mathrm{~nm}$ down to $2.4 \mathrm{~nm}$. We present a model which allows extraction of the important FE parameters such as the coercive fields $\left(E_{C}\right)$, electrostrictive coefficients $\left(Q_{11}\right)$ and spontaneous polarizations $\left(P_{S}\right)$ in the presence of charge injection, storage and leakage with Schottky barriers at the FE oxide-electrode interfaces. We demonstrate FE switching in all the exfoliated B5TFCO nanoflakes. The switching shows very similar characteristics right across the thickness range, and persists in flakes with thicknesses of $2.4 \mathrm{~nm}$ and high aspect ratios (diameter $80 \mathrm{~nm}$ ). This thickness corresponds to just over one-half unit cell or four perovskite oxide blocks plus the associated $\mathrm{Bi}_{2} \mathrm{O}_{2}$ layers. We believe this demonstrates for the first time that the class of selfsupporting 2D oxide materials can include FEs, with all their useful functional properties, greatly increasing the potential range of their applications.

\section{Characterization of Dispersed $\mathrm{Bi}_{5} \mathrm{Ti}_{3} \mathrm{Fe}_{0.5} \mathrm{CO}_{0.5} \mathrm{O}_{15}$ (B5TFCO) Nanoflakes}

B5TFCO has orthorhombic symmetry with lattice parameters $a=0.5373 \mathrm{~nm}, b=0.5430 \mathrm{~nm}$ and $\mathrm{c}=4.1268 \mathrm{~nm}$, as illustrated in Figure $1 .{ }^{[25]}$ Exfoliated nanoflakes were made by ultrasonic exfoliation in a liquid ${ }^{[26]}$ (see Methods) from highly-oriented molten-salt synthesized ceramics. ${ }^{[27]}$ These were dispersed onto Ti-coated Si substrates (see Methods) and characterized using SEM, TEM, AFM and 
PFM. SEM analysis reveals that the dispersed nano-structured flakes have plate-like morphologies,

which is characteristic of the layered Aurivillius-type structures. A range of flake sizes (lateral diameters of $80 \mathrm{~nm}$ to $700 \mathrm{~nm}$ ) can be observed in the SEM images (see Figure S1 for an example) as individual flakes, or stacked on top of each other or overlapped together in clusters. The two larger particles shown in Figure S1 were chosen for FIB cross-sectioning. Cross-sectional HR-TEM images of these (Figure S2) shows that these particles are clusters of flakes. Figure S2 shows the lattice images taken from these. It shows clear evidence for the expected 4-perovskite-block APM structure possessing a unit cell $4.1 \mathrm{~nm}$ long (c-axis), consistent with Figure 1. The TEM data Figure S2 shows that the larger clusters of flakes can be clustered in multi-flake aggregates.. van der Waals forces position the majority of the flakes closely parallel to the substrate surface although not all crystals are aligned perfectly flat. STEM-EDX analysis confirmed the presence of $\mathrm{Bi}$ and $\mathrm{Ti}$ in approximately the correct proportions, with the presence of low levels of Fe and Co as expected from the B5TFCO composition. This, in association with the TEM lattice images, confirms that the larger flakes maintain the APM structure. We will show that the electrical properties of the thicker flakes scale well with thickness, right down to the thinnest flakes. We believe that this is good evidence that the APM structure is maintained down to the thinnest flakes. Figure $\mathbf{2}$ presents evidence that thinner flakes can be found which are not clusters. Here we present (Figure 2a) an AFM topographic image of a single $15 \mathrm{~nm}$ flake of B5TFCO which is lying flat in the substrate. This is nearly circular and approximately 250nm in diameter, giving it a diameter-to-thickness aspect ratio of ca17:1.

Electromechanical responses of the exfoliated B5TFCO flakes were assessed by simultaneous lateral and vertical PFM measurements, respectively, at a single drive frequency of $20 \mathrm{kHz}$. Figure $\mathbf{S 3}$ shows the topography (Figure S3a) of a flake $\sim 300 \mathrm{~nm}$ in diameter and $\sim 33 \mathrm{~nm}$ in height, together with the in-plane (Figure $\mathbf{S} \mathbf{3} \mathbf{b}$ - amplitude, $\mathbf{e}$ - phase) and out-of-plane (Figure $\mathbf{S 3} \mathbf{c}$ - amplitude, $\mathbf{f}$ phase). It is clear from the single frequency PFM images that higher piezoresponses are obtained in the lateral direction $\left(11 \mathrm{pmV}^{-1}\right)$ compared with those measured in the vertical direction $\left(3 \mathrm{pmV}^{-1}\right)$. This is expected, given that the major polarization vector for APMs is along the $a$-axis. ${ }^{[28]}$ The 
enhanced lateral PFM response confirms that the majority of the flakes are preferentially $c$-axis oriented, with their crystallographic a-axes lying in the lateral plane of the film. . Given the relatively low piezoresponses, topography cross-talk is likely also to have some contribution to the single frequency vertical PFM images obtained. In order to amplify the weaker vertical piezoresponse and reduce effects of topography cross-talk, the DART-PFM mode was used (see

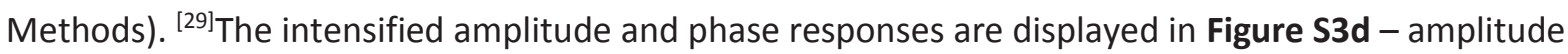
$\boldsymbol{\&} \mathbf{g}$ - phase, respectively. The magnitude of the out-of-plane response is discussed further below. In order to investigate size effects on the electromechanical properties of the exfoliated B5TFCO nanoflakes, vertical DART-PFM imaging was performed on flakes with a range of lateral dimensions ( $230 \mathrm{~nm}$ to $2 \mu \mathrm{m}$ ) and thicknesses ( $14 \mathrm{~nm}$ to $120 \mathrm{~nm}$ ). Figures $2 \mathbf{b} \& \mathbf{c}$ show the vertical amplitude and phase responses from the same flake for which the topography image is shown in Figure 2a. The response appears to be remarkably uniform in both amplitude and phase, and indicative that this flakes is single domain, although evidence for multi-domain structures was observed in other flakes.

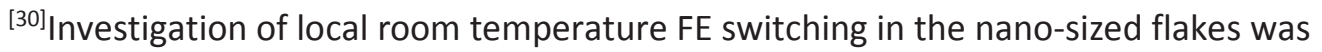
performed by vertical DART-PFM switching spectroscopy measurements at a distinct tip position after the removal of an applied pulsed DC bias ("field off state") (Figure 2). Note that the "field on" loops are not presented here, since electrostrictive effects and electrostatic interactions between the cantilever and the back electrode can complicate the interpretation of the hysteresis loops obtained. However, they are presented and discussed further below. Evidence is presented here for FE hysteresis (Figure $\mathbf{2 d}$ ) and $180^{\circ} \mathrm{FE}$ switching between two antiparallel polarization states (Figure $\mathbf{2 e}$ ) is indicated for flakes from $100 \mathrm{~nm}$ thick down to thicknesses of $14 \mathrm{~nm}$. Vertical DART-PFM switching spectroscopy was also conducted on much-thinner individual flakes, as shown in Figures 3a, b, c. These vertical DART-PFM measurements demonstrate piezoelectricity in nano-structured B5TFCO flakes with thickness of 8nm (which corresponds to 2 unit cells of B5TFCO) and diameter of $160 \mathrm{~nm}$. Note that this flake appears to have two domains (indicated by areas with a $180^{\circ}$ phase shift 
between them) and with a domain wall running approximately vertically down the center of the flake. The presence of different domain structures has previously been demonstrated ${ }^{[30]}$ for nanodots of similar geometry but with irregularities in shape and strain. The multi-domain character may be helping to stabilize the piezoresponse at these smaller dimensions.

Ferroelectricity was investigated for this and other individual flakes: $4.2 \mathrm{~nm}$ thick / $100 \mathrm{~nm}$ diameter (aspect ratio 24:1) and 2.4nm thick / 80nm diameter (aspect ratio 33:1), as demonstrated by the piezoresponse and phase vertical DART-PFM switching spectroscopy hysteresis loops (Figure 3d,e respectively). $180^{\circ} \mathrm{FE}$ switching is observed in all cases. The smaller flake thicknesses correspond to one unit-cell and to just over half a unit cell of B5TFCO, respectively. Note that one edge of the $4.2 \mathrm{~nm}$ thick flake has overlapped on top of the $2.4 \mathrm{~nm}$ flake and is therefore somewhat tilted. These flakes were initially imaged in contact mode AFM (no AC bias) to obtain the topography images inset in Figure 3d,e. Following this, DART-PFM switching spectroscopy hysteresis loops were obtained. PFM imaging (i.e. scanning the PFM tip while applying an AC drive voltage) of the smaller flakes ( $4.2 \mathrm{~nm}$ and $2.4 \mathrm{~nm}$ in height) could not be obtained because the process caused physical damage. Large-scale surface damage at increased bias has previously been reported for PZT $\left(\mathrm{PbZr}_{0.52} \mathrm{Ti}_{0.48} \mathrm{O}_{3}\right)$ nanodots. ${ }^{[31]}$

APMs are well-known FEs and ferroelectricity has been established for Co-substituted $\mathrm{Bi}_{5} \mathrm{Ti}_{3} \mathrm{FeO}_{15}$ ceramics and thin film samples. ${ }^{[27,32]}$ However, the fact that ferroelectricity has been established for larger structures is not proof that ferroelectricity will exist at smaller dimensions. We now consider the results of the PFM experiments to ascertain if they support FE behavior, considering that other contributions (e.g. electrostatic effects, electrostrictive effects, surface electrochemical reactions, charge migration effects) $)^{[33]}$ can affect the PFM signals. We note that the loops follow the typical counterclockwise behavior expected for standard FEs. ${ }^{[33]}$ The piezoresponse versus applied voltage PFM loops for the B5TFCO flakes were performed in a pulsed DC mode which minimizes electrostatic and electrostrictive contributions to the signal ${ }^{[34]}$. However, measurement of loop evolution with $V_{a c}$ (drive amplitude) is a necessary step to establish the validity of PFM 
hysteresis measurements. ${ }^{[35]}$ For most electromechanically active materials with dominant piezoelectric coupling and weak electrostrictive contributions, when below the coercive voltage of the material, the piezoresponse amplitude is directly proportional to $V_{a c}$ while the piezoresponse phase is $V_{a c}$ independent. ${ }^{[36], ~[33]}$ Linearity of piezoresponse $(p m)$ with drive amplitude $\left(V_{a c}\right)$ was verified for a flake of $76 \mathrm{~nm}$ height and $579 \mathrm{~nm}$ diameter at a constant DC bias of $15 \mathrm{~V}$ (Figure S4).

\section{PFM Response Modelling}

An interesting aspect to the hysteresis loops observed from the exfoliated flakes and shown in Figures $\mathbf{2}$ and $\mathbf{3}$ is their shape at high voltages. The piezoresponse is observed to increase at high voltage, rather than to saturate, as would be expected for a perfect switched FE. The shape is reproducible across the series of flake thicknesses and applies to both flake aggregates and single crystals. This type of loop shape has been reported before from a range of materials, including PFM data from thin films of e.g. $\mathrm{BiFeO}_{3}{ }^{[37]}$ strain-free $\mathrm{SrTiO}_{3}{ }^{[38]}$ and $\mathrm{P} / \mathrm{E}$ loops from PVDF-TrFE films and composites with $5 \% \mathrm{BiFeO}_{3}-\mathrm{NaNbO}_{3}$ powders, ${ }^{[39]}$ although hitherto it has not been explained. It is likely that the effect observed is due to the injection of charge from the electrodes into the FE after the switching of polarization in both positive and negative senses, creating a layer of trapped, but mobile, charge (electrons or holes) that increases the local polarization and thus the piezoresponse. However, when the field is removed the charge might be expected to leak away, and this is what we observe.

To understand the detailed shape of the PFM loops (and, indeed the whole of the field-on, field-off response), we need to consider in detail how the PFM experiment is performed. Starting from zero applied field, a small AC voltage is applied to the PFM tip, and the piezoresponse is measured in individual time slots [ ]t (e.g. [t $\mathrm{t}=0.5 \mathrm{~ms}) . \mathrm{N}$ (say $\mathrm{N}=50$ ) readings are taken at zero applied bias, giving a total field-off measurement time of at $\mathrm{t}=\mathrm{N}$. [ $\mathrm{t}$ ( $25 \mathrm{~ms}$ in this example). This is the first "field-off" response measurement. A DC bias $\left(V_{1}\right)$ is applied for a time at (in this example for $25 \mathrm{~ms}$ ) and a further N (50) "field-on" response measurements are taken. The DC bias is removed and a further N (50) "field-off" response measurements taken. These are the "field-off" piezoresponse 
readings corresponding to $V_{1}$, with the readings being averaged over a pre-set number of the 50 readings taken. This process is repeated with $V_{1}$ increased (or decreased) by an amount a $\mathrm{V}$, to give the full hysteresis loop. The parameters $[\mathrm{t}, \mathrm{N}$ and $\mathrm{Q} \mathrm{V}$ can all be varied within the PFM instrument parameters. To model the piezoresponse behavior in the presence of mobile charge injection and leakage, consider how the charge will move into and out of the FE during the field-on and field-off periods, taking account of the switching of the FE polarization through the loop cycle. To do this, we use the approach of Pintilie and Alexe ${ }^{[40]}$, later modified by Fan et al. ${ }^{[41]}$, who considered the thermionic emission of current into an insulator across a metal-insulator junction, including the effects of FE polarization and charge trapping. The descriptions are based on a modified version of the Schottky barrier model.

The energy bands at a Schottky contact between a metal and an insulator (or semiconductor) are illustrated as in Figure $\mathbf{4 a}^{[41]} . \phi_{B}^{0}$ is Schottky barrier height set by the Fermi level in the metal, $V_{b i}$ is the height of the built-in potential barrier and $w$ is the width of the Schottky depletion region. In Figure $\mathbf{4 b}$ we see the description of the barrier in the presence of the FE polarization $P$. The FE is assumed to have been poled by the application of a negative voltage to the metal, so the FE polarization can be treated as a sheet of positive charge situated at a depth $\delta$ below the metal-FE interface. This is called "negatively poled" and denoted $P \uparrow$ and changes (in this case increases) $V_{b i}$ to some value $V_{b i}^{\prime}$ and the $\phi_{B}^{0}$ is increased to $\phi_{A p p}$. The converse poled case is shown in Figure 4c. The layer defined by $\delta$ is sometimes referred to as the "dead layer". Fan et al. ${ }^{[1]}$ assume that charges injected into the FE become trapped and reside in the dead layer with a volumetric density $N_{\text {Trapped }}$ to give a total charge $Q_{\text {Trapped }}$, (in $\mathrm{Cm}^{-2}$ ) given by:

$$
Q_{\text {Trapped }}=q N_{\text {Trapped }} \delta
$$

Where $q$ is the electron charge. 
The following equations are derived by Fan et al. ${ }^{[41]}$ They are very similar to the equations derived by Pintilie and Alexe ${ }^{[40]}$ but include the effects of the charge trapped in the dead layer.

$V_{b i}^{\prime}=V_{b i} \mp \frac{P}{\varepsilon_{0} \varepsilon_{s t}} \delta \pm \frac{q N_{\text {Trapped }}}{2 \varepsilon_{0} \varepsilon_{s t}} \delta^{2}$

$V_{b i}=\phi_{b}^{0}-\frac{k_{B} T}{q} \ln \left[\frac{N_{V}}{p(T)}\right]$

In the above, $\varepsilon_{0}=$ permittivity of free space; $\varepsilon_{s t}=$ low frequency permittivity of $\mathrm{FE}, k_{B}=$ Boltzmann's Constant; $N_{V}=$ Density of states for holes in the valence band; $p(T)=$ Hole concentration at temperature $T$.

The depletion width is given by:

$w=\left(\frac{2 \varepsilon_{0} \varepsilon_{s t}\left(V+V_{b i}^{\prime}\right)}{q N_{e f f}}\right)^{\frac{1}{2}}$

Here, $V=$ applied voltage and $N_{e f f}=$ effective charge density in the films.

The maximum field at the interface is given by:

$E_{m}=\frac{q N_{\text {eff }} w}{\varepsilon_{0} \varepsilon_{s t}} \pm \frac{P}{\varepsilon_{0} \varepsilon_{s t}} \mp \frac{q N_{\text {Trapped }}}{2 \varepsilon_{0} \varepsilon_{S t}} \delta$

In equations (2) and (5), the lower signs apply to the case of a negatively poled FE where $P \uparrow$ and the upper signs for the reverse case.

This can be put into a form that eliminates $w$ :

$E_{m}=\left[\frac{2 q N_{e f f}\left(V+V_{b i}^{\prime}\right)}{\varepsilon_{0} \varepsilon_{s t}}\right]^{\frac{1}{2}} \pm \frac{P}{\varepsilon_{0} \varepsilon_{s t}} \mp \frac{q N_{\text {Trapped }}}{2 \varepsilon_{0} \varepsilon_{s t}} \delta$

The apparent barrier height is given by:

$\phi_{A p p}=\phi_{B}^{0}-\sqrt{\frac{q E_{m}}{4 \pi \varepsilon_{0} \varepsilon_{o p t}}}$

Where $\varepsilon_{\text {opt }}=$ optical frequency permittivity of FE

In the presence of strong carrier recombination, Pintilie ${ }^{[40]}$ quotes: 
$J=q N_{V} v_{D} \exp \left(\frac{-q \phi_{A p p}}{k_{B} T}\right)$

Where $v_{D}$ is the carrier diffusion velocity which is proportional to the carrier drift mobility $\mu_{D}$. This can be rewritten as:

$J=q N_{C} \mu_{D} E_{m} \exp \left(\frac{-q \phi_{A p p}}{k_{B} T}\right)$

Where $N_{C}$ is the density of carriers available for the drift into the charge traps. This is likely to be mediated via a hopping process so that the current density is "bulk limited", rather than being limited by thermionic emission into the FE from the electrodes.

Based on the previous observation that $\mu_{D}$ is strongly dependent on temperature, we assume that $\mu_{D}$ is determined by some sort of thermal activation process and an equation of the form:

$\mu_{D}=\mu_{D}^{o} \exp \left(\frac{A}{k_{B} T}\right)$

To model the space charge contribution to the PFM response, we assume that during the "field-on" part of the PFM cycle, some space charge is injected into the sample, with a current as determined by the Schottky barrier model described above. This adds to the sheet polarization due to the FE polarization. During the "field-off" part of the cycle, the space charge will tend to leak away with a certain time constant that will depend on the electrical conductivity of the system, permittivity etc. This will give an exponential-decay function. We assume that in each part of the field-on cycle, the charge is injected to give a trapped charge:

$Q_{\text {Trapped }}=J \Delta t$

Where $\Delta t=$ "field-on" time as defined above.

This will add to the total polarization $P_{T}$ in the system, so that:

$P_{T}=P+Q_{\text {Trapped }}$

During a field-off cycle, the magnitude of the piezoelectric coefficient $d_{e f f}$ is then given in terms of the electrostrictive coefficient $Q_{11}$ by: 
$d_{\text {eff }}=2 Q_{11} P_{T} \varepsilon_{0} \varepsilon_{s t}$

During a "field-on" cycle, the applied voltage induces a further polarization $P_{\text {ind }}$, which is given by:

$P_{\text {ind }}=V \kappa \varepsilon_{e f f} \varepsilon_{o} / t$

Where $\kappa$ is a variable introduced to allow for effects of FE thickness, tip geometry etc. on the induced polarization.

During the field-on cycles, the sign of $P_{i n d}$ is observed to be opposite to the sign of $P_{T}$.

$d_{\text {eff }}=2 Q_{11}\left(P_{T}-P_{\text {ind }}\right) \varepsilon_{0} \varepsilon_{s t}$

In making a calculation for $d_{e f f}$, we also need to take account of the fact that the "deadlayers" at each FE-metal interface are non-FE, low permittivity materials. These place two capacitors in series with the active FE layer, which reduces the AC field across the FE, and hence the measured $d_{\text {eff }}$, by a factor $F_{\text {Red }}$ :

$F_{\text {Red }}=\left(\frac{\varepsilon_{s t}}{\varepsilon_{\text {opt }}} \frac{1}{\left(\frac{t}{2 \delta}-1\right)}+1\right)^{-1}$

We also need to take account of the way the trapped charge leaks away during both the field-on and field-off cycles. This accomplished by applying an exponential charge leakage term. To model the time structure, we split each field-on and field-off period into a number of $\mathrm{N}$ steps (e.g. 50). We assume that the injected current is constant throughout each step period. (This is an approximation as the current is affected to some extent by the number of trapped charges.) The $N_{\text {Trapped }}$ (and hence the value of $Q_{\text {Trapped }}$ ) at the end of each period $\Delta t$ is calculated in the following way:

1. The $N_{\text {Trapped }}$ is given a starting value for $\mathrm{V}=0$, say $N_{\text {Trapped }}^{0}$. This is chosen to be equal to the value at the end of a complete cycle when the voltage again returns to zero. It is found that the result is not strongly dependent on the value chosen for $N_{\text {Trapped }}^{0}$.

2. During each section $\mathrm{m}$ of a field-on period, the value of $N_{\text {Trapped }}$ is calculated as: 
$N_{\text {Trapped }}^{m}=\frac{J \Delta t}{q \delta}+N_{\text {Trapped }}^{m-1} \exp (-\gamma \Delta t)$

Where $\gamma$ is a decay constant and $N_{\text {Trapped }}^{m-1}$ is the number of carriers trapped at the end of the previous period. $J$ is calculated using equations (6) and (9) with the value of $N_{\text {Trapped }}$ in equation (6) being set as the value of $N_{\text {Trapped }}^{m-1}$

3. During each section $\mathrm{m}$ of a field-off period, the value of $N_{\text {Trapped }}$ at the end of each section $\mathrm{m}$ is calculated as:

$N_{\text {Trapped }}^{m}=N_{\text {Trapped }}^{m-1} \exp (-\gamma \Delta t)$

The value of $N_{\text {Trapped }}^{m}$ at the end of each period is used to calculate the trapped charge density from:

$Q_{\text {Trapped }}=N_{\text {Trapped }}^{m} q \delta$

This is then used in equations (12) to (15) to calculate $d_{e f f}$ throughout the field-on and fieldoff cycles.

In their modelling, Fan et al. ${ }^{[41]}$ used a square hysteresis loop. Real FEs are almost-never so well-behaved, and in this model the values of the FE polarization as a function of field used in Equations (5) and (6) are derived using the semi-empirical tanh function described by Miller et al. ${ }^{[42]}$ and Blom et al. ${ }^{[43]}$ Here, the polarization $P(E)$ is described as a function of the electric field $E$ using:

$P(E)=\varepsilon_{0}\left(\varepsilon_{o p t}-1\right) E+P_{S} \tanh \left[\frac{E \pm E_{C}}{2 \alpha}\right]$

Where $P_{S}=$ Spontaneous Polarization, $P_{R}=$ Remanent Polarization, $E_{C}=$ Coercive Field and:

$\alpha=E_{c}\left[\ln \frac{1+\beta}{1-\beta}\right] \quad \beta=\frac{P_{R}}{P_{S}}$

This produces a loop as illustrated in Figure S4. In this graph, the "positive going" arm of the loop takes the minus-sign in the tanh function in Equation (18), while the "negative going" arm of the loop takes the plus-sign. Loop shifts (either in the polarization or voltage axes) caused by in-built biases (charge or voltage asymmetries) are frequently observed in FE thin films (Refs). In this model we have allowed for the possibility of loop displacements in either the voltage or polarization axes by introducing the parameters $P_{\text {Shift }}$ and $V_{\text {Shift }}$. 
In our model, we use the following parameters for all the examples:

$N_{V}=N_{C}=2 \times 10^{28} \mathrm{~m}^{-3} ; N_{e f f}=4 \times 10^{28} \mathrm{~m}^{-3} p(300)=5 \times 10^{24} \mathrm{~m}^{-3} ; \Phi_{B}^{0}=1.3 \mathrm{eV}$ (these are similar to the values used by Fan et al. for PZT films $\left.{ }^{[41]}\right) . N_{\text {trapped }}(t=0)=10^{21} \mathrm{~m}^{-3}$

The low frequency permittivies $\varepsilon_{s t}$ have been measured for a range of Aurivillius oxides, and the values are quite similar. These include 140 for $\mathrm{CaBi}_{4} \mathrm{Ti}_{4} \mathrm{O}_{15}{ }^{[44]}$, and 160 for hot-forged $\mathrm{Bi}_{4} \mathrm{Ti}_{3} \mathrm{O}_{12}{ }^{[45]}$. A value of $\varepsilon_{s t}=140$ has been used here. A refractive index of 2.7 in $\mathrm{Bi}_{4} \mathrm{Ti}_{3} \mathrm{O}_{12}$ has been measured by Cummins $^{[46]}$, corresponding to an optical permittivity of 7.3. We have used a value of $\varepsilon_{\text {opt }}=6.5$ in this model. The value of $\delta$ (dead layer thickness) was fixed at $0.035 \mathrm{~nm}$, similar to the value derived by Dawber et al. ${ }^{[4]}$ in their modelling of dead layer thicknesses and polarization field correction in FE switching in PVDF, and the value fits well with our observations.

The parameters $V_{C}$ (coercive voltage), $V_{\text {Shift }}, P_{S}, \Delta P_{R}=P_{S}-P_{R}, P_{\text {Shift }}, \mu_{D}$ (carrier drift mobility), $\gamma$ (charge decay constant), $\varepsilon_{\text {eff }}$ (effective permittivity) and $Q_{11}$ (electrostrictive coefficient) were fitted parameters. These were fitted by a process of least-squares (minimizing $\left.r^{2}=\sum\left(d_{o b s}-d_{c a l c}\right)^{2} / \sum\left(d_{o b s}^{2}\right)\right)$ over the full field-on and field-off data for measurements of $\mathrm{d}_{\mathrm{eff}}$. Two data points were ignored on each side of the voltage switching transient, so 46 data points were fitted in each field-on or field-off period, and the models were fitted over one-and-one-half full voltage cycles so nearly 10,000 data points were taken for each fitting. Note that the first half-cycle was ignored as this serves to polarize the point on the flake in a particular direction. It was observed that $Q_{11}=2( \pm 0.1) \times 10^{-2} \mathrm{~m}^{4} \mathrm{C}^{-2}$. This is in the middle of the range observed for FE perovskite titanates $^{[48]}$.

FE and butterfly loops were plotted by taking the average of the last $10 d_{\text {eff }}$ values in each field-off period, for both the observed and modelled values and plotting them against the applied voltage value in the immediately-preceding field-on period.

The results of the fitting for a 30nm thick flake are shown in Figure 6. It can be seen that the model describes the behaviour of $d_{\text {eff }}$ well, in both the time-structure and around the butterfly and 
hysteresis loops. The parameters derived from the fitting process, and the $r^{2}$ values for each fit, are given in Table S1.

Figure 7 shows comparisons between the observed and modelled PFM piezoresponse data for flakes of 2.4, 4.2, 8 and $14 \mathrm{~nm}$ thickness, together with the time-structure PFM response for the 2.4nm flake, including an example where we have "zoomed-in" on part of the time structure. The agreement between the modelled and observed data is very good for both the PFM loops and the field-on and field-off time structures. If we look in detail at the materials parameters used to model the data, we find that the low frequency $\left(\varepsilon_{s t}\right)$ and optical $\left(\varepsilon_{\text {opt }}\right)$ permittivities used (140 and 6.5) are in excellent agreement with parameters for similar materials in the literature: $\varepsilon_{s t}=140$ for $\mathrm{CaBi}_{4} \mathrm{Ti}_{4} \mathrm{O}_{15}{ }^{[44]}$, and 160 for hot-forged $\mathrm{Bi}_{4} \mathrm{Ti}_{3} \mathrm{O}_{12}{ }^{[45]}$ and $\varepsilon_{\text {opt }}=7.5$ in $\mathrm{Bi}_{4} \mathrm{Ti}_{3} \mathrm{O}_{12}\left(C u m m i n s{ }^{[46]}\right)$. The value of $\delta$ (dead layer thickness) used in our model was fixed at $0.035 \mathrm{~nm}$. This is similar to the value derived by Dawber et al. ${ }^{[47]}$ in their modelling of dead layer thicknesses and polarization field correction in FE switching in PVDF. We observed that $Q_{11}=2( \pm 0.1) \times 10^{-2} \mathrm{~m}^{4} \mathrm{C}^{-2}$. This is in the middle of the range observed for FE perovskite titanates ${ }^{[48]}$. Based on an out-of-plane spontaneous polarization of $0.04 \mathrm{Cm}^{-2}$ observed by Takenaka ${ }^{[45]}$ in hot-forged $\mathrm{Bi}_{4} \mathrm{Ti}_{3} \mathrm{O}_{12}, \varepsilon_{s t}=160$ and a $Q_{11}=2 \times 10$ ${ }^{2} \mathrm{~m}^{4} \mathrm{C}^{-2}$ we calculate for that material an out-of-plane piezoelectric coefficient $d$ of $2.3 \mathrm{pmV}^{-1}$. This is in excellent agreement with the values obtained at zero applied voltage for the $14 \mathrm{~nm}$ thick flake shown in Figure $\mathbf{4 d}$. The thinner flakes show somewhat smaller values of $d$. The parameters extracted from the fitting of the model to the observed data on all the flakes studies are given in Table S1. The values of $P_{S}$ are not strongly dependent on flake thickness, showing a good deal of scatter, which is what would be expected if some of the flakes were slightly canted out of the plane. We observe an average value of $0.04( \pm 0.02) \mathrm{Cm}^{-2}$. The mean value is in very good agreement with the value observed for $\mathrm{Bi}_{4} \mathrm{Ti}_{3} \mathrm{O}_{12}$, as reported above, although this may simply be a coincidence, as discussed below. 
We do not see a strongly systematic variation of either the drift mobility (average $\mu_{D}=1.4 \times 10^{-6} \mathrm{~m}^{2} \mathrm{~V}^{-1} \mathrm{~s}^{-1}$ ) or the charge decay constant (average $\gamma=18 \mathrm{~s}^{-1}$ ) with flake thickness (see Table S1). The dependence of these parameters on temperature, and the comparison of flakes with thin films, will be the subject of a further publication.

Figure 7a shows the variation of the coercive field $E_{C}$ with thickness. (Here, we have taken the mean values of $E_{C}$ for all the $33 \mathrm{~nm}$ thickness measurements conducted at different maximum applied voltages $V_{\text {Max }}$ ). Empirically, $E_{C}$ increases with decreasing thickness of the FE material ${ }^{[47]}$. As explained by Ducharme et al. ${ }^{[49]}$ the extrinsic coercive field observed in FEs is caused by localized nucleations of domains with reversed polarization. Defects are often the source of domain walls, and thicker flakes have a higher probability of containing defects, meaning that thicker flakes are more easily polarized than thinner flakes. As first observed by Kay and Dunn in triglycine sulfate ${ }^{[50]}$, the scaling of $E_{C}$ with thickness $(t)$ follows the semi-empirical scaling law: $E_{C} \propto t^{-\frac{2}{3}}$. In the case of our data, if we simply plot $\left(E_{C}\right)$ vs $t$ (using a log-log scale) we observe an empirical relationship $E_{C} \propto t^{-1}$ (the "uncorrected" data in Figure 7a). However, if we apply a depolarization correction to the field data, as discussed by Dawber et al. ${ }^{[47]}$, such that:

$E_{C}($ Corrected $)=E_{C} /\left(t+2 \delta \varepsilon_{s t}\right)$ where $t$ is the thickness of the relevant flake, and 0 the thickness of the dead layer (using the model values of $0=0.035 \mathrm{~nm}$ and ${ }_{\mathrm{st}}=140$ ), then we get the "corrected" plot in Figure 7a, which produces an exponent of -0.7, close to the value of 0.66 expected from the Kay and Dunn law.

Finally, we should comment on the observed values of $\kappa$ and the way these are observed to vary with flake thickness. In a perfect system, we would expect $\kappa=1$, so that the polarization induced by the applied field is directly proportional to the field and a constant value of $\varepsilon_{s t}$ for all flake thicknesses, but this is not the case here. The observed values of $\kappa$ are plotted as a function of flake thickness in Figure $\mathbf{7 b}$. Empirically, we observe that $\kappa$ is proportional to flake thickness over the full range of flake thicknesses, although there is a fair degree of scatter in the measurements. There are 
a range of reasons as to why this might be the case. The effect of field concentration at the PFM tip and the way that interacts with the flake thickness may be one cause of non-linearity and the effects of damage in the oxide at low thicknesses may be another. This is an area for further study.

\section{Discussion and Outlook}

We have reported here the fabrication and characterization by various probe microscopies of exfoliated APM B5TFCO nanoflakes. These are either stacked together as multi-flakes or dispersed individually with flake thicknesses ranging from $120 \mathrm{~nm}$ to $2.4 \mathrm{~nm}$. PFM imaging has demonstrated piezoelectricity in both lateral and vertical directions with higher piezoresponses in the lateral $\left(11 \mathrm{pmV}^{-1}\right)$ compared with the vertical $\left(2-3 \mathrm{pmV}^{-1}\right)$, as expected for APMs with the major polarization vector along the $a$-axis. Local PFM switching loops were generated for the nano-sized flakes. The details of the PFM response, including the time-structure in both field-on and field-off states, has been accurately described with a model using charge injection through a Schottky barrier at the electrode-FE oxide interface, in association with charge storage in (and time-dependent leakage from) a surface layer, where the charge injection is affected by the magnitude and sign of the FE polarization. The parameters derived from this model such as permittivities, electrostrictive coefficients, spontaneous polarization magnitudes etc. are all in excellent agreement with values for similar materials taken from the literature. Based on the parameters derived from fitting this model to the data, the coercive field $\left(E_{c}\right)$ scales with thickness $(t)$, following the semi-empirical scaling law: $E_{C} \propto t^{-\frac{2}{3}}$. It is believed that the balance of evidence obtained from the PFM measurements indicates that room temperature ferroelectricity can exist, and can be switched, in nano-structured B5TFCO flakes as thin as 4.2 and $2.4 \mathrm{~nm}$. The observation of multi-domain structures within single, unpoled nanoflakes which have not undergone PFM-driven switching is supporting evidence for the existence of ferroelectricity in these very thin flakes. The observation of out-of-plane FE switching in such thin flakes for an APM with $m=4$ is very interesting. Normally, we would not expect to see any component of $P_{S}$ out of the basal plane of an APM structure for which $m$ is even. ${ }^{[28,51]}$ Clearly, we see a switchable component of $P_{s}$ in our measurements. The most-likely explanation is that the 
flakes are not lying perfectly parallel to the underlying substrate surface, in which case we would reasonable expect to be able to switch a component of the in-plane polarization. A simple estimate shows that for an in-plane $P_{S}$ of $0.4 \mathrm{Cm}^{-2}$ (as expected for most APM FEs), a flake would only need to be sitting at an angle $(\Phi)$ of $<6^{\circ}$ to give an out-of-plane component of $\approx 0.04 \mathrm{Cm}^{-2}$, the average value observed in this work. However, the smallest measured values of out-of-plane $P_{s}$ in Table S1 would correspond to values of $\Phi \approx 2.5^{\circ}$ and the largest $\Phi \approx 10^{\circ}$. Nevertheless, the observed values of the out-of-plane $P_{S}$ component observed in this work are remarkably consistent from grain to grain, and across the different grain thicknesses, and very similar to the value observed for $\mathrm{Bi}_{4} \mathrm{Ti}_{3} \mathrm{O}_{12}$, so the intriguing possibility remains that we are seeing an intrinsic out-of-plane component for $P_{S}$, which would mean that the symmetry is not as expected $\left(\mathrm{A} 2_{1} \mathrm{am}\right),{ }^{[52]}$ but something lower. This possibility requires further work to resolve. It should be noted that a thickness of $4.2 \mathrm{~nm}$ corresponds closely to the thickness which would be expected for a single unit cell, while $2.4 \mathrm{~nm}$ is a thickness corresponding to half the unit cell, plus one $\mathrm{Bi}_{2} \mathrm{O}_{2}$ layer, or slightly more than half a unit-cell of B5TFCO (see Figure 1). The thinnest flake would thus appear to be four perovskite blocks in thickness, sandwiched between two $\mathrm{Bi}_{2} \mathrm{O}_{2}$ layers. We believe that these are the first PFM measurements on exfoliated APMs. The results are a noteworthy contribution to the fundamental research of both ultra-thin piezoelectric and FE materials and also demonstrate for the first time that wide band-gap oxide FEs can be added to the growing class of 2D materials. This is an important conclusion, as it offers the prospect of incorporating the wide range of useful FE properties, vis: a large switchable, built-in spontaneous polarization, piezoelectricity, pyroelectricity etc. into future electronic devices exploiting 2D materials. In addition, we believe that the model for PFM response presented here will be a useful tool for those working with this technique as a means for characterizing FE behavior in thin materials.

\section{Methods}


Highly-oriented polycrystalline ceramics of $\mathrm{Bi}_{5} \mathrm{Fe}_{0.5} \mathrm{Co}_{0.5} \mathrm{Ti}_{3} \mathrm{O}_{15}$ prepared via molten salt synthesis ${ }^{[27]}$ were used as the starting material for the liquid exfoliation process. $\mathrm{Bi}_{5} \mathrm{Ti}_{3} \mathrm{Fe}_{0.5} \mathrm{Co}_{0.5} \mathrm{O}_{15}$ powder was dispersed in an aqueous solution of the surfactant sodium cholate $\left(0.1 \mathrm{mgml}^{-1}\right.$ concentration) using an ultrasonic processor (Sonics VX-750) operating at 75\% amplitude for 90 mins. The dispersions were left to settle for 72 hours. The top 50\% was then decanted into 2 glass vials and centrifuged (Hettick Mickro 22R) at 500rpm for 30 mins to remove any remaining unexfoliated material. Decantation was carried out by pipetting off the top $50 \%$ of each dispersion in a single glass vial. Thin films were made by vacuum filtration immediately after centrifugation. Dispersions were filtered through a nitrocellulose membrane (pore size $25 \mathrm{~mm}$ ) and the deposited films were washed with $400 \mathrm{ml}$ of Millipore water to remove surfactant. Films were dried for 20 minutes at $50^{\circ} \mathrm{C}$ before transfer onto $\mathrm{Ti}$ coated $\mathrm{SiO}_{2}$. Films were wetted with isopropyl alcohol and pressed against the substrate. The cellulose filter was removed by treatment with acetone vapor and subsequent acetone liquid baths. Films were annealed at $800^{\circ} \mathrm{C}$ to remove any remaining surfactant. Flake morphology was analyzed by scanning electron microscopy (SEM) (Zeiss Ultra Plus SEM and FEI DualBeam Helios NanoLab 600i). Cross-sections of the flakes were prepared for micro-structural analysis using a FEI DualBeam Helios NanoLab 600i SEM/FIB (focused ion beam) (final thinning at 93pA 30kV, final polish 2kV 28pA). Samples were gold-coated to prevent charging. Micro-structural analysis was performed on the B5TFCO flakes using HR-TEM (high resolution transmission electron microscopy; Jeol 2100 transmission electron microscope; 200kV; double tilt holder). Elemental mapping was performed using STEM-EDX (Scanning Transmission Electron Microscopy-Energy Dispersive X-Ray Diffraction; Helios Nanolab STEM - medium spot size, $\mathbf{x}$-ray generation area 10-30 $\mathrm{nm}$ in diameter, 100-300 nm lamella thickness). A commercial atomic force microscope (AFM) (MFP-3D ${ }^{\mathrm{TM}}$, Asylum Research) was used for topography mapping of the films. Diameter and height values reported in this manuscript were determined by topographical imaging. Electromechanical responses of the films were measured by PFM using an Asylum Research MFP-3D ${ }^{\mathrm{TM}}$ AFM in contact mode, equipped with a HVA220 Amplifier for PFM using Single Frequency (drive frequency of 20 
$\mathrm{kHz}$ ) PFM. The angular torsion of the cantilever as it oscillates was monitored in lateral

measurements, which were conducted in a similar manner to the vertical measurements except the 'InFast' option was programmed to 'Lateral'. The Dual AC Resonance Tracking Piezoresponse Force Microscopy (DART-PFM) ${ }^{[29]}$ mode was used to boost the vertical piezo signal. In this mode, the PFM signal is measured at the tip-sample contact resonance frequency, where the drive frequencies are adjusted as the probe scans over the changing sample topography in order to reduce topographical cross-talk. Olympus AC240TM Electrilevers, Ti/Pt coated silicon cantilevers (Al reflex coated, $70 \mathrm{kHz}$ resonant frequency, $\sim 320 \mathrm{kHz}$ contact resonance frequency) were used for PFM and topography imaging. The Inverse Optical Lever Sensitivity of the cantilevers was calibrated according to the MFP-3D Procedural Operation 'Manualette', the system inherent background was determined using a non-piezoelectric silicon wafer and the PFM was then calibrated using $\alpha$-quartz as a reference sample. Vertical hysteresis loop measurements were obtained at fixed tip positions on the surface of the particles by switching spectroscopy PFM (SS-PFM) ${ }^{[53]}$ using a triangular step waveform (comprised of pulse DC (direct current) bias voltage of 15-35V and an AC (alternating current) drive voltage $\left(V_{a c}\right)$ of $\left.5.5 \mathrm{~V}\right)$. The waveform was cycled twice at a frequency of $0.3 \mathrm{~Hz}$ with $55-83 \mathrm{AC}$ steps per waveform. Hysteresis loops taken on a particular flake location were repeated at least three times whereupon repeatable results were obtained. 


\section{Author Contributions}

R.W.W. proposed the idea of exfoliating APMs into nano-structured flakes and exploring their FE properties. M.P. and A.J.B. synthesized the B5TFCO ceramic sample. R.J.S. and J.N.C. developed the liquid exfoliation method and fabricated the nano-structured B5TFCO flakes. M.S. performed HRSEM, HR-TEM and STEM-EDX characterization of the B5TFCO flakes. L.K. performed the PFM measurements. R.W.W. devised the model for the PFM response and undertook the modelling of the data. L.K. and R.W.W. wrote the first draft of the manuscript. The final version of the manuscript was written through contributions of all authors. All authors have given approval to the final version of the manuscript.

\section{Notes:}

The authors declare no competing financial interest.

\section{Funding Sources}

The support of Science Foundation Ireland (SFI) under the FORME Strategic Research Cluster Award number $07 /$ SRC/I1172 is gratefully acknowledged. This publication has emanated from research conducted with the financial support of the Royal Society and Science Foundation Ireland (SFI) University Research Fellowship UF 140263. 


\section{References}

[1] a) R. Mas-Ballesté, C. Gómez-Navarro, J. Gómez-Herrero, F. Zamora, Nanoscale 2011, 3, 20; b) A. C. Ferrari, F. Bonaccorso, V. Fal'ko, K. S. Novoselov, S. Roche, P. Bøggild, S. Borini, F. H. L. Koppens, V. Palermo, N. Pugno, J. A. Garrido, R. Sordan, A. Bianco, L. Ballerini, M. Prato, E. Lidorikis, J. Kivioja, C. Marinelli, T. Ryhänen, A. Morpurgo, J. N. Coleman, V. Nicolosi, L. Colombo, A. Fert, M. Garcia-Hernandez, A. Bachtold, G. F. Schneider, F. Guinea, C. Dekker, M. Barbone, Z. Sun, C. Galiotis, A. N. Grigorenko, G. Konstantatos, A. Kis, M. Katsnelson, L. Vandersypen, A. Loiseau, V. Morandi, D. Neumaier, E. Treossi, V. Pellegrini, M. Polini, A. Tredicucci, G. M. Williams, B. Hee Hong, J.-H. Ahn, J. Min Kim, H. Zirath, B. J. van Wees, H. van der Zant, L. Occhipinti, A. Di Matteo, I. A. Kinloch, T. Seyller, E. Quesnel, X. Feng, K. Teo, N. Rupesinghe, P. Hakonen, S. R. T. Neil, Q. Tannock, T. Löfwander, J. Kinaret, Nanoscale 2015, 7, 4598.

[2] V. Nicolosi, M. Chhowalla, M. G. Kanatzidis, M. S. Strano, J. N. Coleman, Science 2013, 340.

[3] X. Dong, M. Osada, H. Ueda, Y. Ebina, Y. Kotani, K. Ono, S. Ueda, K. Kobayashi, K. Takada, T. Sasaki, Chemistry of Materials 2009, 21, 4366.

[4] B.-W. Li, M. Osada, T. C. Ozawa, R. Ma, K. Akatsuka, Y. Ebina, H. Funakubo, S. Ueda, K. Kobayashi, T. Sasaki, Japanese Journal of Applied Physics 2009, 48, 09 KA15.

[5] M. Osada, T. Sasaki, Journal of Materials Chemistry 2009, 19, 2503.

[6] P. Poizot, S. Laruelle, S. Grugeon, L. Dupont, J. M. Tarascon, Nature 2000, 407, 496.

[7] V. Subramanian, H. Zhu, B. Wei, Journal of Power Sources 2006, 159, 361.

[8] Y. Li, G. Chen, C. Zhou, Z. Li, Catalysis Letters 2008, 123, 80.

[9] Y. Yu, L. Ma, P. Cai, R. Zhong, C. Ye, J. Shen, G. D. Gu, X. H. Chen, Y. Zhang, Nature 2019, 575, 156.

[10] R. W. Whatmore, in Springer Handbook of Electronic and Photonic Materials, DOI: 10.1007/978-0-387-29185-7_27, Springer-Verlag, Boston 2007, p. 597.

[11] J. F. Scott, C. A. Paz de Araujo, Science 1989, 246, 1400.

[12] Q. Su, P. Kirby, E. Komuro, M. Imura, Q. Zhang, R. Whatmore, IEEE Transactions on Microwave Theory and Techniques 2001, 49, 769.

[13] a) R. W. Whatmore, Reports on Progress in Physics 1986, 49, 1335; b) P. Muralt, Reports on Progress in Physics 2001, 64, 1339.

[14] a) S. Palto, L. Blinov, A. Bune, E. Dubovik, V. Fridkin, N. Petukhova, K. Verkhovskaya, S. Yudin, Ferroelectrics 1996, 184, 127; b) L. M. Blinov, V. M. Fridkin, S. P. Palto, A. V. Sorokin, S. G. Yudin, Thin Solid Films 1996, 284-285, 474.

[15] A. Zenkevich, M. Minnekaev, Y. Lebedinskii, K. Bulakh, A. Chouprik, A. Baturin, R. Mantovan, M. Fanciulli, O. Uvarov, Thin Solid Films 2012, 520, 4586.

[16] F. Xue, W. Hu, K.-C. Lee, L.-S. Lu, J. Zhang, H.-L. Tang, A. Han, W.-T. Hsu, S. Tu, W.-H. Chang, C.-H. Lien, J.-H. He, Z. Zhang, L.-J. Li, X. Zhang, Advanced Functional Materials 2018, 28, 1803738.

[17] S. Yuan, X. Luo, H. L. Chan, C. Xiao, Y. Dai, M. Xie, J. Hao, Nature Communications 2019, 10, 1775.

[18] J. J. Gervacio-Arciniega, E. Prokhorov, F. J. Espinoza-Beltrán, G. Trapaga, Journal of Applied Physics 2012, 112, 052018.

[19] B. Aurivillius, Ark. Kemi. 1949, 1, 499.

[20] E. C. Subbarao, Physical Review 1961, 123, 2202.

[21] a) C. A. P. de Araujo, J. D. Cuchiaro, L. D. McMillan, M. C. Scott, J. F. Scott, Nature 1995, 374, 627; b) B. H. Park, B. S. Kang, S. D. Bu, T. W. Noh, J. Lee, W. Jo, Nature 1999, 401, 682.

[22] N. A. Lomanova, I. V. Pleshakov, M. P. Volkov, V. V. Gusarov, Materials Science and Engineering: B 2016, 214, 51. 
[23] a) L. Keeney, T. Maity, M. Schmidt, A. Amann, N. Deepak, N. Petkov, S. Roy, M. E. Pemble, R. W. Whatmore, Journal of the American Ceramic Society 2013, 96, 2339; b) A. Faraz, T. Maity, M. Schmidt, N. Deepak, S. Roy, M. E. Pemble, R. W. Whatmore, L. Keeney, Journal of the American Ceramic Society 2017, 100, 975; c) L. Keeney, C. Downing, M. Schmidt, M. E. Pemble, V. Nicolosi, R. W. Whatmore, Scientific Reports 2017, 7, 1737; d) M. Schmidt, A. Amann, L. Keeney, M. E. Pemble, J. D. Holmes, N. Petkov, R. W. Whatmore, Scientific Reports 2014, 4, 5712; e) S. Manipatruni, D. E. Nikonov, C.-C. Lin, T. A. Gosavi, H. Liu, B. Prasad, Y.-L. Huang, E. Bonturim, R. Ramesh, I. A. Young, Nature 2019, 565, 35.

[24] a) V. Chevalller, G. Nihoul, V. Madigou, Journal of Solid State Chemistry 2008, 181, 439; b) S. Ida, C. Ogata, U. Unal, K. Izawa, T. Inoue, O. Altuntasoglu, Y. Matsumoto, Journal of the American Chemical Society 2007, 129, 8956; c) J.-Y. Kim, I. Chung, J.-H. Choy, G.-S. Park, Chemistry of Materials 2001, 13, 2759; d) M. B. Sreedhara, B. E. Prasad, M. Moirangthem, R. Murugavel, C. N. R. Rao, Journal of Solid State Chemistry 2015, 224, 21.

[25] X. Mao, W. Wang, X. Chen, Y. Lu, Applied Physics Letters 2009, 95, 082901.

[26] R. J. Smith, P. J. King, M. Lotya, C. Wirtz, U. Khan, S. De, A. O'Neill, G. S. Duesberg, J. C. Grunlan, G. Moriarty, J. Chen, J. Wang, A. I. Minett, V. Nicolosi, J. N. Coleman, Advanced Materials 2011, 23, 3944.

[27] M. Palizdar, T. P. Comyn, M. B. Ward, A. P. Brown, J. P. Harrington, S. Kulkarni, L. Keeney, S. Roy, M. E. Pemble, R. W. Whatmore, C. Quinn, S. H. Kilcoyne, A. J. Bell, Journal of Applied Physics 2012, 112, 073919.

[28] T. Watanabe, H. Funakubo, Journal of Applied Physics 2006, 100.

[29] B. J. Rodriguez, C. Callahan, S. V. Kalinin, R. Proksch, Nanotechnology 2007, 18, 475504.

[30] B. J. Rodriguez, X. S. Gao, L. F. Liu, W. Lee, I. I. Naumov, A. M. Bratkovsky, D. Hesse, M. Alexe, Nano Letters 2009, 9, 1127.

[31] B. J. Rodriguez, S. Jesse, M. Alexe, S. V. Kalinin, Advanced Materials 2008, 20, 109.

[32] L. Keeney, S. Kulkarni, N. Deepak, M. Schmidt, N. Petkov, P. F. Zhang, S. Cavill, S. Roy, M. E. Pemble, R. W. Whatmore, Journal of Applied Physics 2012, 112, 052010.

[33] R. K. Vasudevan, N. Balke, P. Maksymovych, S. Jesse, S. V. Kalinin, Applied Physics Reviews 2017, 4, 021302.

[34] S. Jesse, H. N. Lee, S. V. Kalinin, Review of Scientific Instruments 2006, 77, 073702.

[35] E. Strelcov, Y. Kim, J. C. Yang, Y. H. Chu, P. Yu, X. Lu, S. Jesse, S. V. Kalinin, Applied Physics Letters 2012, 101, 192902.

[36] a) H. Bo, Y. Kan, X. Lu, S. Peng, X. Wang, Y. Liu, W. Cai, R. Xue, J. Zhu, Review of Scientific Instruments 2010, 81, 043704; b) S. V. Kalinin, B. J. Rodriguez, S. Jesse, J. Shin, A. P. Baddorf, P. Gupta, H. Jain, D. B. Williams, A. Gruverman, Microscopy and Microanalysis 2006, 12, 206.

[37] B. Kim, F. P. Barrows, Y. Sharma, R. S. Katiyar, C. Phatak, A. K. Petford-Long, S. Jeon, S. Hong, Scientific Reports 2018, 8, 203.

[38] H. W. Jang, A. Kumar, S. Denev, M. D. Biegalski, P. Maksymovych, C. W. Bark, C. T. Nelson, C. M. Folkman, S. H. Baek, N. Balke, C. M. Brooks, D. A. Tenne, D. G. Schlom, L. Q. Chen, X. Q. Pan, S. V. Kalinin, V. Gopalan, C. B. Eom, Physical Review Letters 2010, 104, 197601.

[39] R. P. Ummer, R. B, C. Thevenot, D. Rouxel, S. Thomas, N. Kalarikkal, RSC Advances 2016, 6, 28069.

[40] L. Pintilie, M. Alexe, Journal of Applied Physics 2005, 98, 124103.

[41] Z. Fan, H. Fan, Z. Lu, P. Li, Z. Huang, G. Tian, L. Yang, J. Yao, C. Chen, D. Chen, Z. Yan, X. Lu, X. Gao, J.-M. Liu, Physical Review Applied 2017, 7, 014020.

[42] S. L. Miller, R. D. Nasby, J. R. Schwank, M. S. Rodgers, P. V. Dressendorfer, Journal of Applied Physics 1990, 68, 6463.

[43] P. W. M. Blom, R. M. Wolf, J. F. M. Cillessen, M. P. C. M. Krijn, Physical Review Letters 1994, 73, 2107.

[44] K. W. Kwok, H. Y. Wong, Journal of Physics D: Applied Physics 2009, 42, 095419.

[45] T. Takenaka, K. Sakata, Journal of Applied Physics 1984, 55, 1092. 
[46] S. E. Cummins, L. E. Cross, Journal of Applied Physics 1968, 39, 2268.

[47] M. Dawber, P. Chandra, P. B. Littlewood, J. F. Scott, Journal of Physics: Condensed Matter 2003, 15, L393.

[48] K. Uchino, L. E. Cross, R. E. Newnham, S. Nomura, Phase Transitions 1980, 1, 333.

[49] S. Ducharme, V. M. Fridkin, A. V. Bune, S. P. Palto, L. M. Blinov, N. N. Petukhova, S. G. Yudin, Physical Review Letters 2000, 84, 175.

[50] H. F. Kay, J. W. Dunn, The Philosophical Magazine: A Journal of Theoretical Experimental and Applied Physics 1962, 7, 2027.

[51] a) R. E. Newnham, R. W. Wolfe, J. F. Dorrian, Materials Research Bulletin 1971, 6, 1029; b) H. Irie, M. Miyayama, Applied Physics Letters 2001, 79, 251.

[52] C. H. Hervoches, A. Snedden, R. Riggs, S. H. Kilcoyne, P. Manuel, P. Lightfoot, Journal of Solid State Chemistry 2002, 164, 280.

[53] a) S. Jesse, A. P. Baddorf, S. V. Kalinin, Appl. Phys. Lett. 2006, 88, 062908; b) S. Jesse, H. N. Lee, S. V. Kalinin, Vol. 77, AIP, 2006, 073702. 
Figures

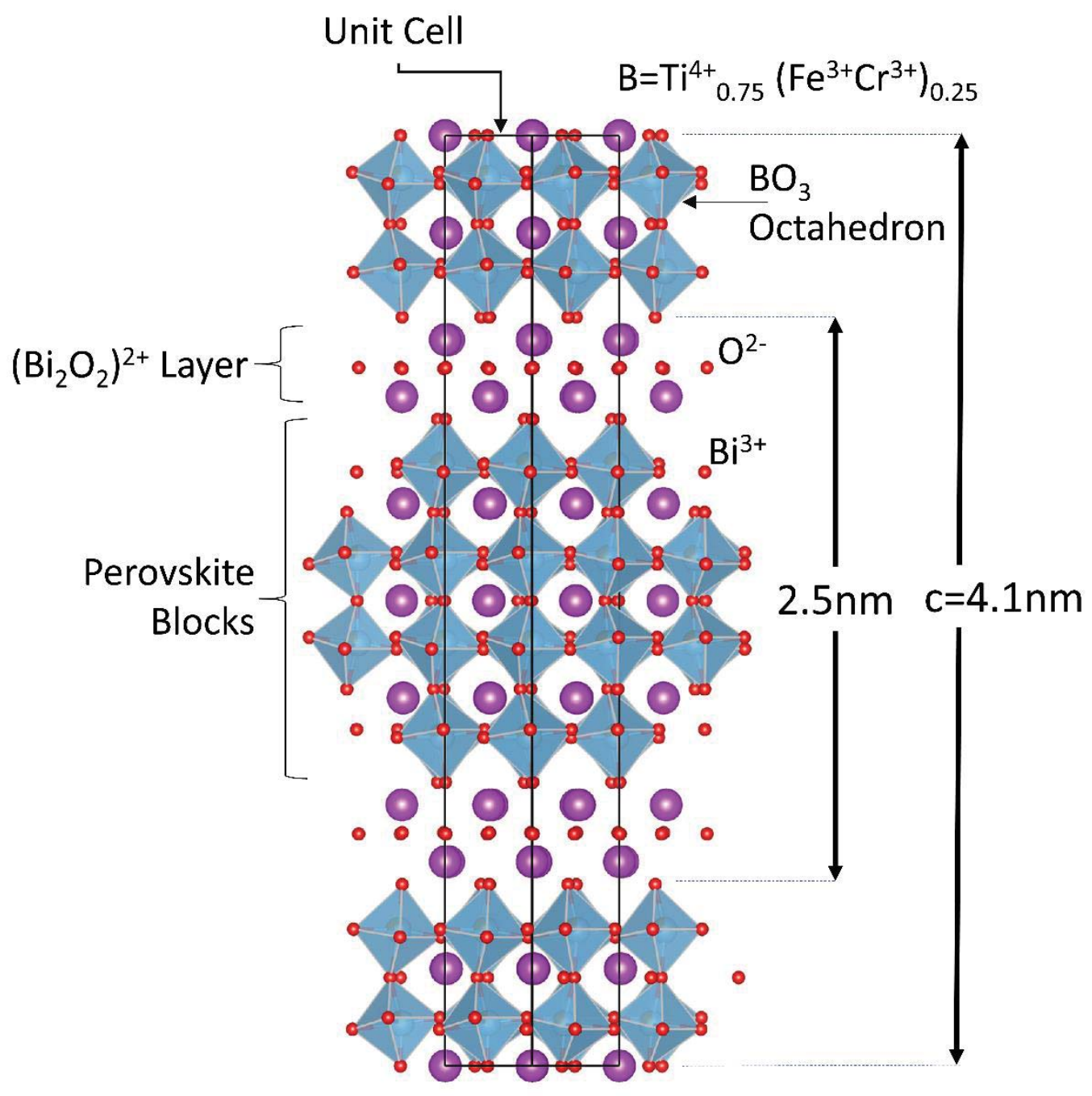

Figure 1: Schematic diagram of the B5TFCO APM structure, viewed approximately along [110], illustrating the main structural features and the relevant unit cell dimensions. 
Figure 2. Representative a. topography, $\mathbf{b}$. vertical DART-PFM amplitude and $\mathbf{c}$. vertical DART-PFM phase images of a single $15 \mathrm{~nm}$-high flake of exfoliated B5TFCO. Vertical DART-PFM switching spectroscopy $\mathbf{h}$ piezoresponse and i phase loops of exfoliated B5TFCO nanoflakes at room temperature after removal of an applied DC bias. 
Figure 3. Representative a topography, $\mathbf{b}$ vertical DART-PFM amplitude and $\mathbf{c}$ vertical DART-PFM phase images of an individual exfoliated 8nm thick B5TFCO flake. Vertical DART-PFM switching spectroscopy $\mathbf{d}$ piezoresponse and e phase loops of individual exfoliated B5TFCO flakes at room temperature after removal of an applied DC bias. The topography of flakes with height $2.4 \mathrm{~nm}$ and $4.2 \mathrm{~nm}$ are shown in the inset in $\mathbf{d}$ and the topography of flake with height $8 \mathrm{~nm}$ is shown in the inset in e. 

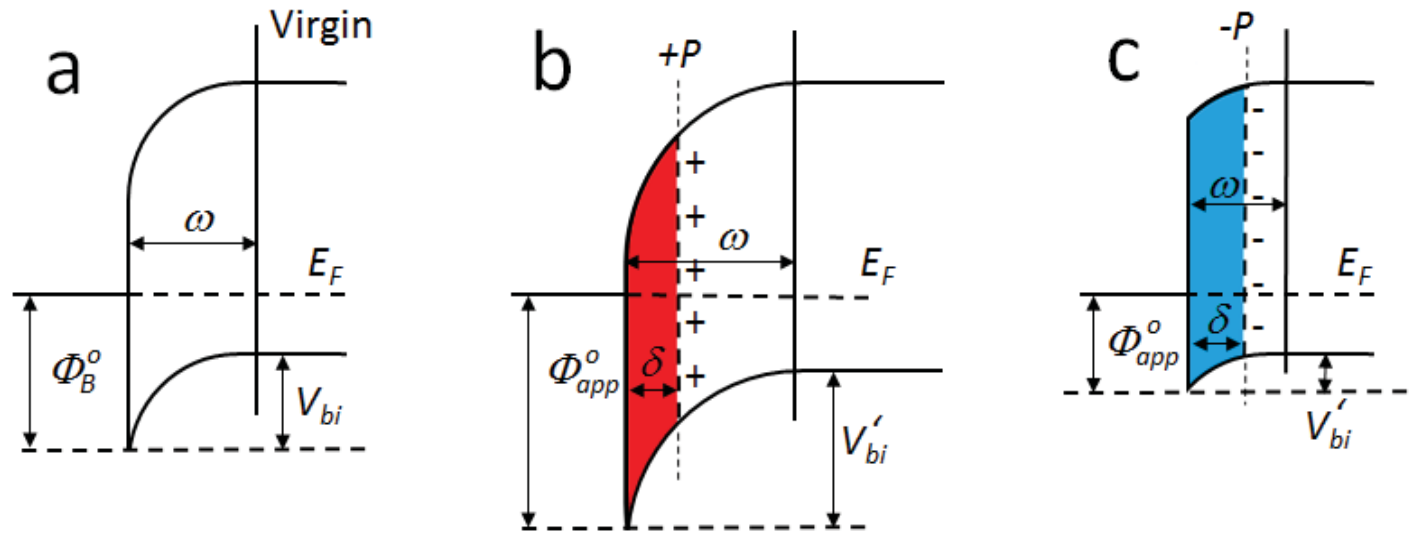

Figure 4. Energy band diagrams of a Schottky diode in a virgin (without polarization and trapped charges), b negatively poled and c positively poled states (redrawn with permission from Fan et al. 2017, American Physical Society $\left.{ }^{[41]}\right)$.

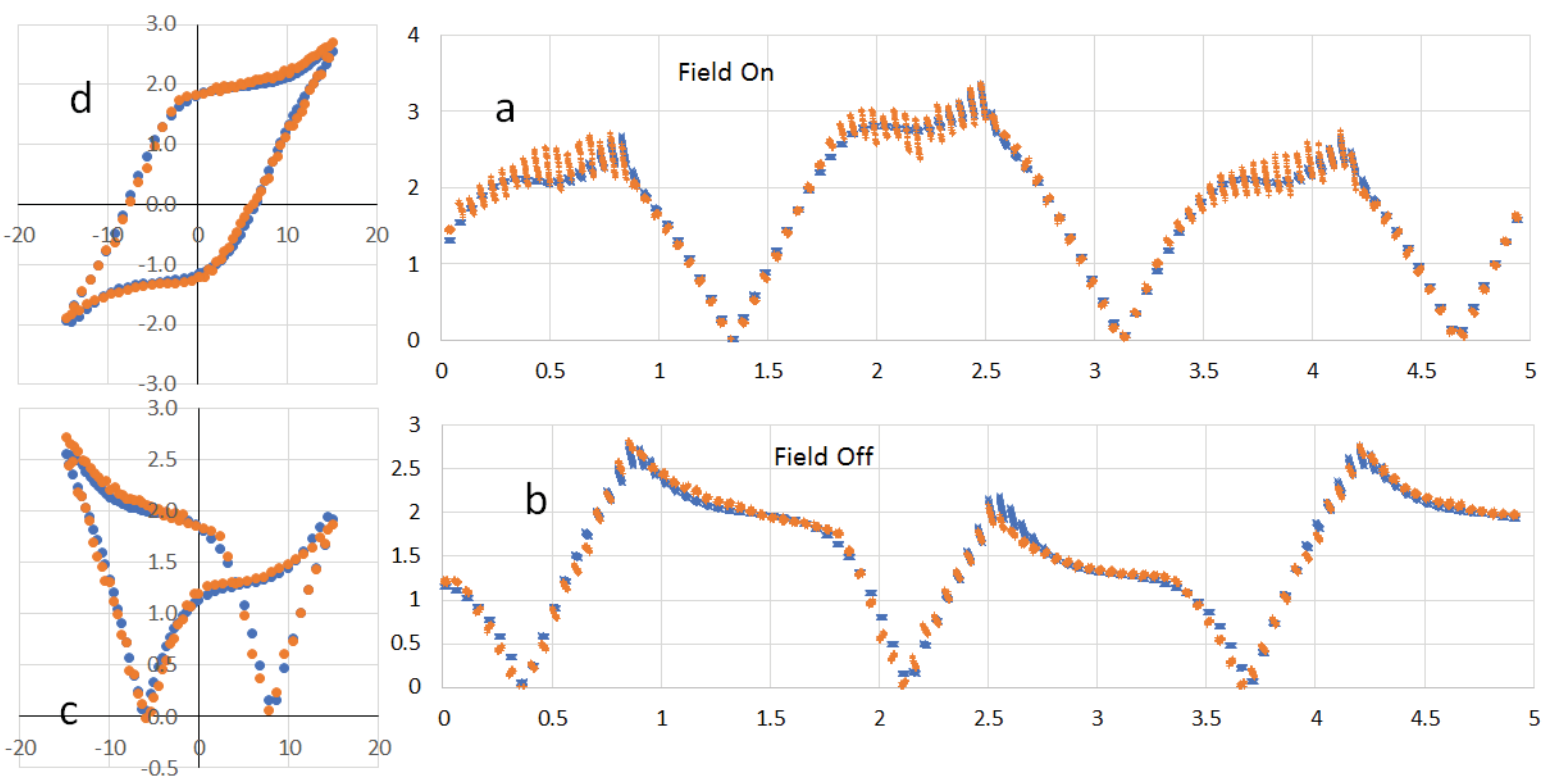

Figure 5. Results of fitting the parameters as discussed in the text for a 30nm thick B5TFCO flake.

This shows a Field-on and $\mathbf{b}$ Field-off time responses in $\mathrm{pmV}^{-1}$. In each case the horizontal axes are plotted in seconds. c Butterfly and $\mathbf{d}$ Hysteresis loops in $\mathrm{pmV}^{-1}$ plotted vs applied voltage. In each case, the observed data is plotted in orange and the modelled data in blue. 

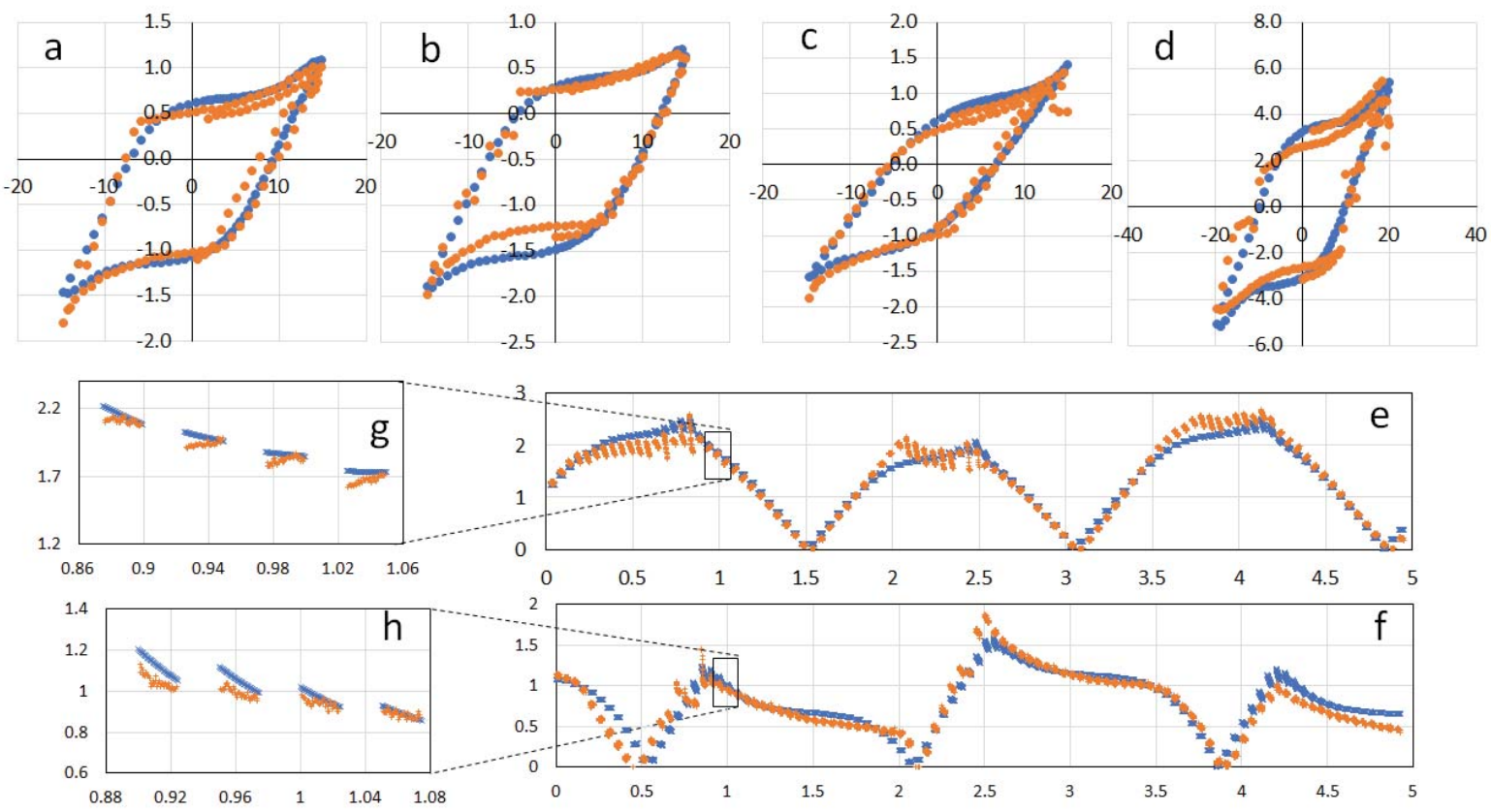

Figure 6. Comparison of measured and modelled PFM piezoresponse hysteresis loops for a $2.4 \mathrm{~nm}, \mathbf{b}$ $4.2 \mathrm{~nm}, \mathbf{c} 8 \mathrm{~nm}$ and $\mathbf{d} 14 \mathrm{~nm}$ exfoliated B5TFCO flakes. In each case the piezoresponse (vertical axes) is measured in $\mathrm{pmV}^{-1}$ and the applied voltage (horizontal axes) in volts. Figures $\mathbf{3}$ e and $\mathbf{f}$ show respectively the field-on and field-off time structures of the piezoresponse signals (in pmV ${ }^{-1}$ ) for the $2.4 \mathrm{~nm}$ flake. Figures $\mathbf{3} \mathbf{g}$ and $\mathbf{h}$ show respectively magnified sections of the field-on and field-off time structures of the piezoresponse signals $\left(\mathrm{in}_{\mathrm{pmV}}{ }^{-1}\right.$ ) for the $2.4 \mathrm{~nm}$ flake. The horizontal axes are plotted in seconds. In each case, the observed data is plotted in orange and the model data is plotted in blue. 

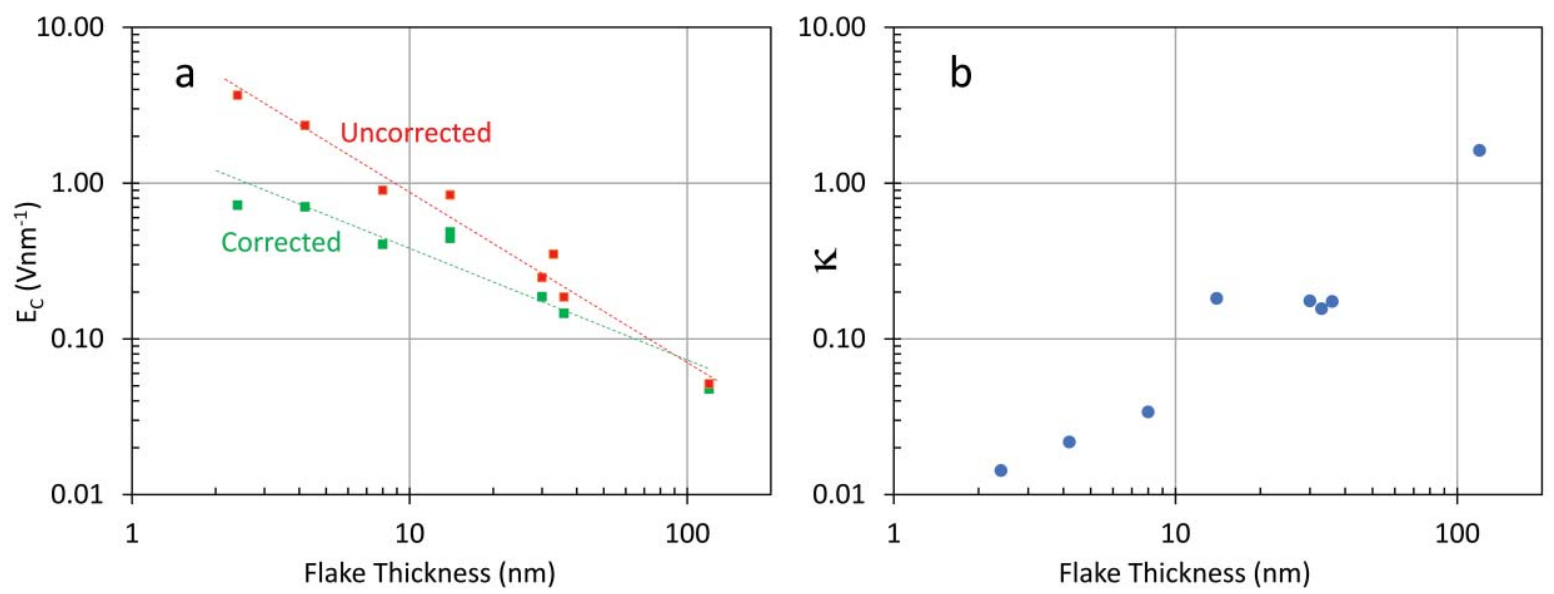

Figure 7. $\mathbf{a}$ Log-log plots of the uncorrected and corrected coercive fields vs flake thickness and $\mathbf{b}$ Log-log plot of the variable $\mathrm{k}$ vs flake thickness as derived from the fits of the model to the measured piezoresponse data. 


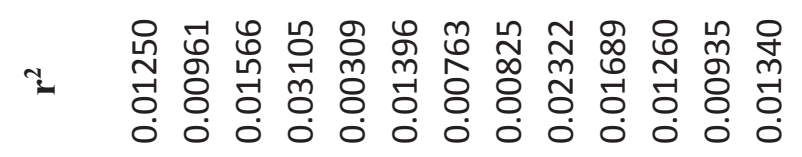

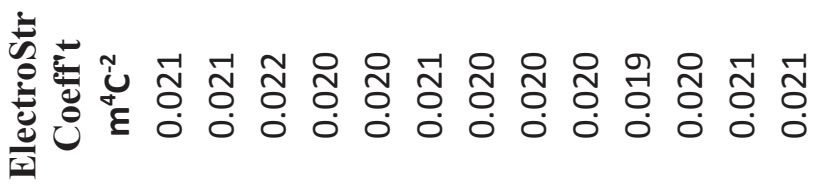

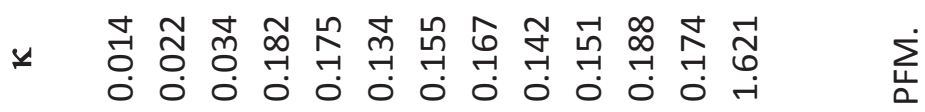

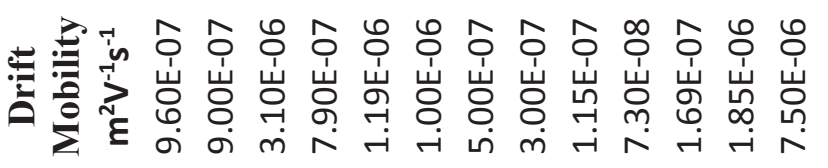

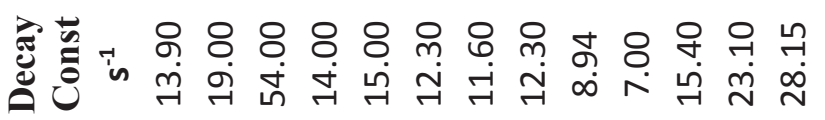

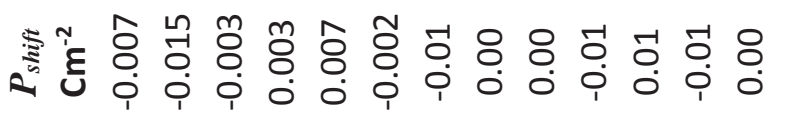

₹

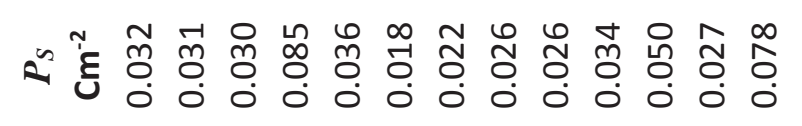

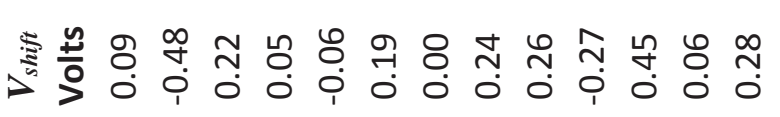

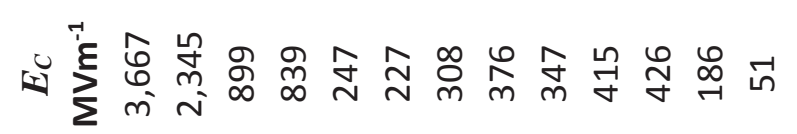

>

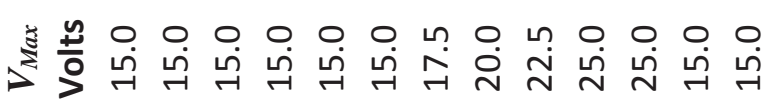

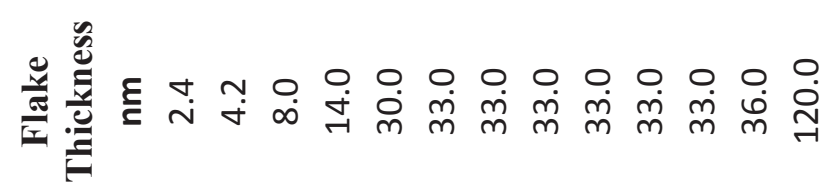


Ferroelectric Behavior in Exfoliated 2D Aurivillius Oxide Flakes of Sub-Unit Cell Thickness

Supporting Information

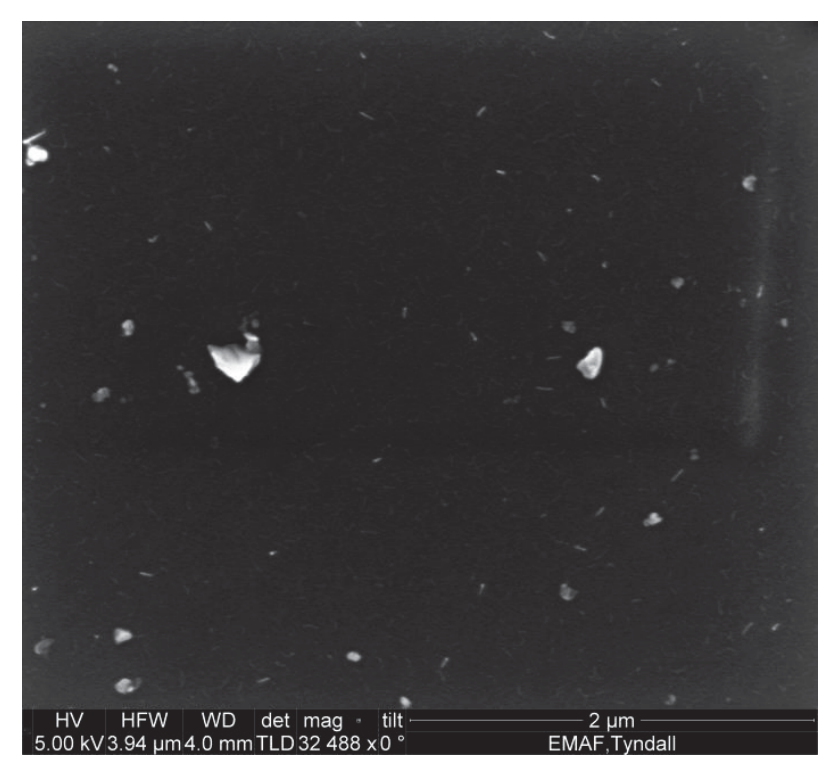

Figure S1. Supporting Information: Representative top-view HRSEM image of exfoliated B5TFCO flakes. 


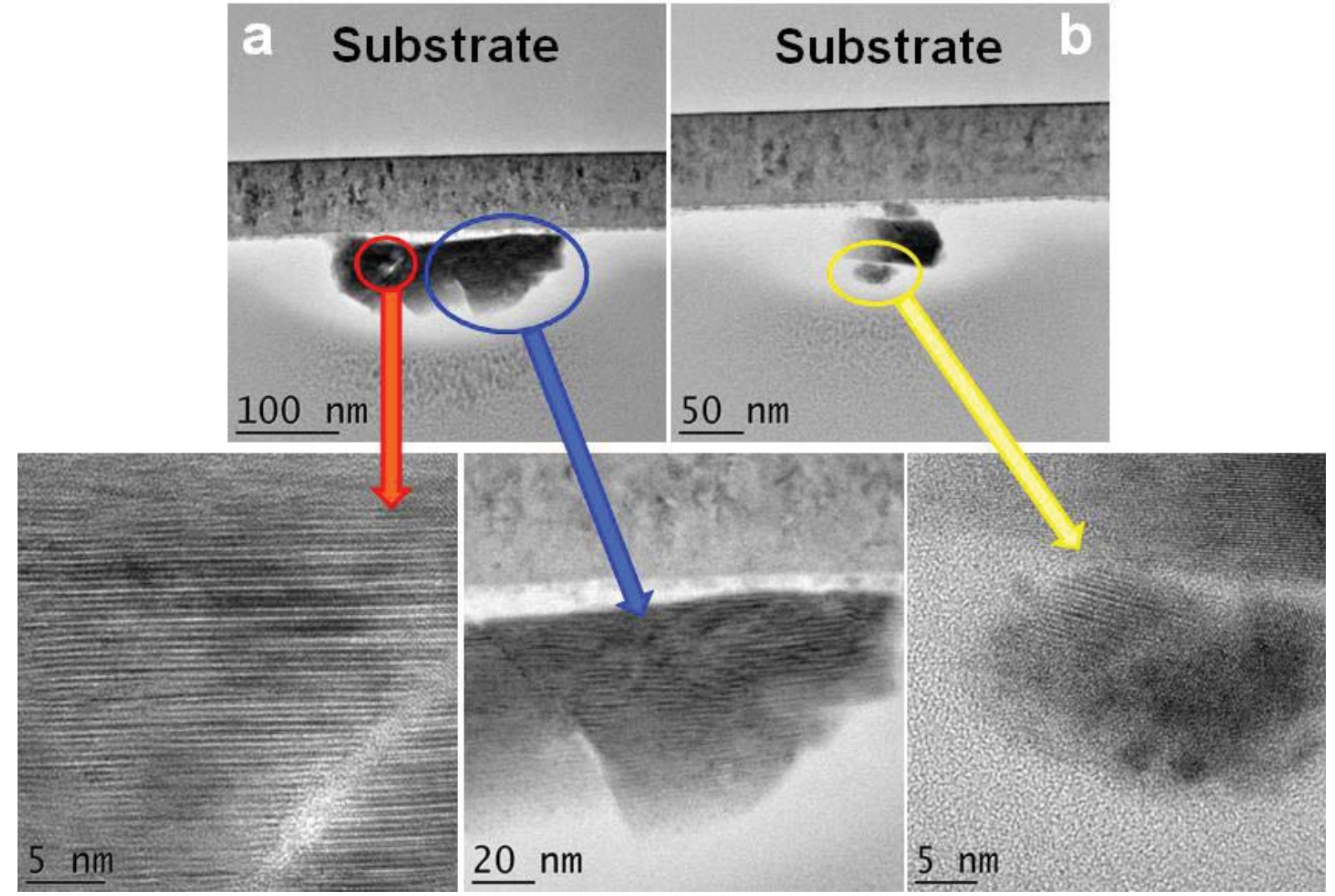

Figure S2. Cross-sectional TEM images of exfoliated B5TFCO flakes. HRTEM of a larger horizontal cluster of multi-flakes (maximum thickness of $80 \mathrm{~nm}$ and diameter of $230 \mathrm{~nm}$ ) a and HRTEM of a smaller vertical multi-flake stack (maximum thickness of $65 \mathrm{~nm}$ and diameter of $80 \mathrm{~nm}$ ) b. 

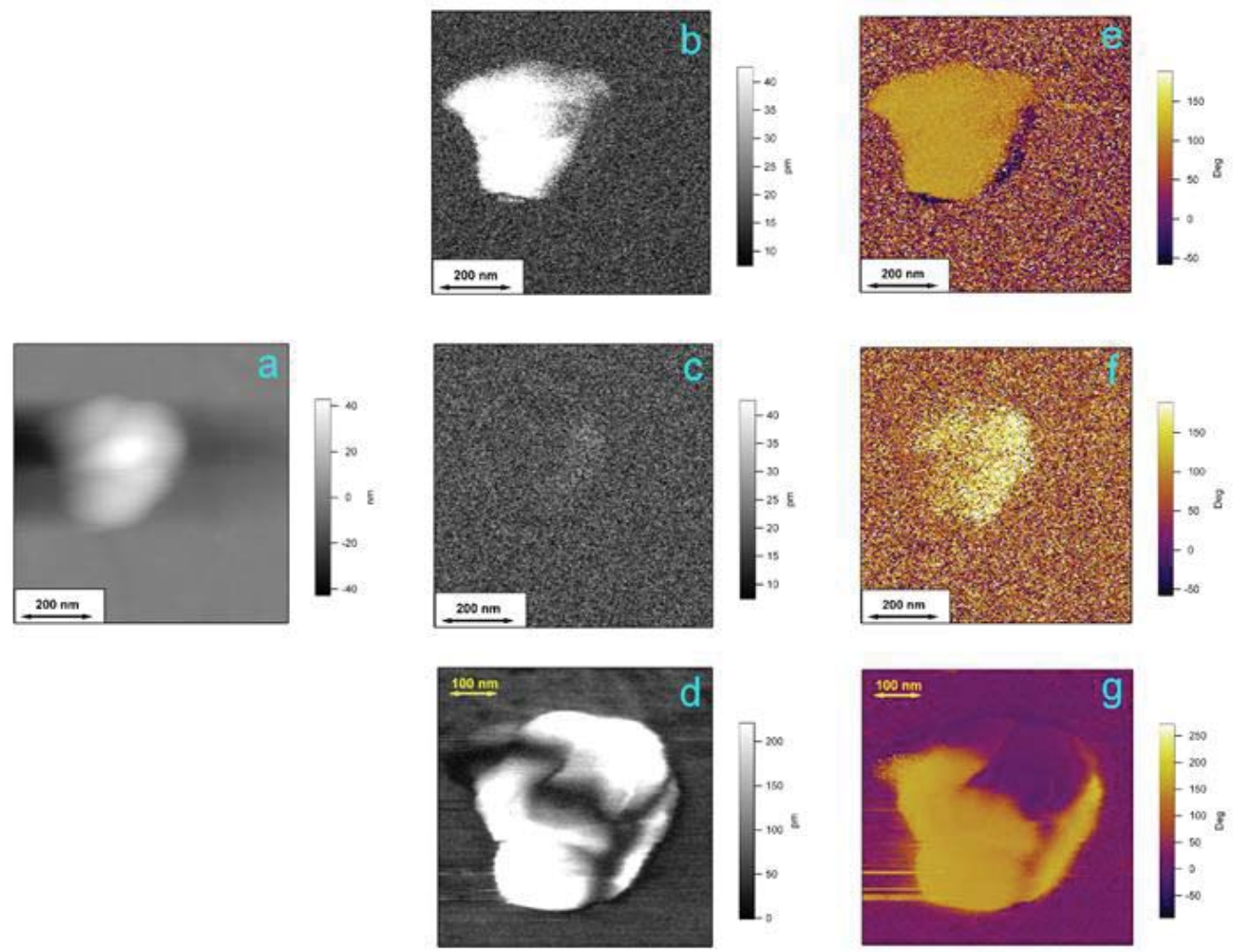

Figure S3. Representative a. topography, b. lateral single frequency PFM amplitude, e. lateral single frequency PFM phase, c. vertical single frequency PFM amplitude and f. vertical single frequency PFM phase $\mathbf{d}$. vertical DART-PFM amplitude and $\mathbf{g}$. vertical DART-PFM phase images of a multi-flake cluster of exfoliated B5TFCO. 


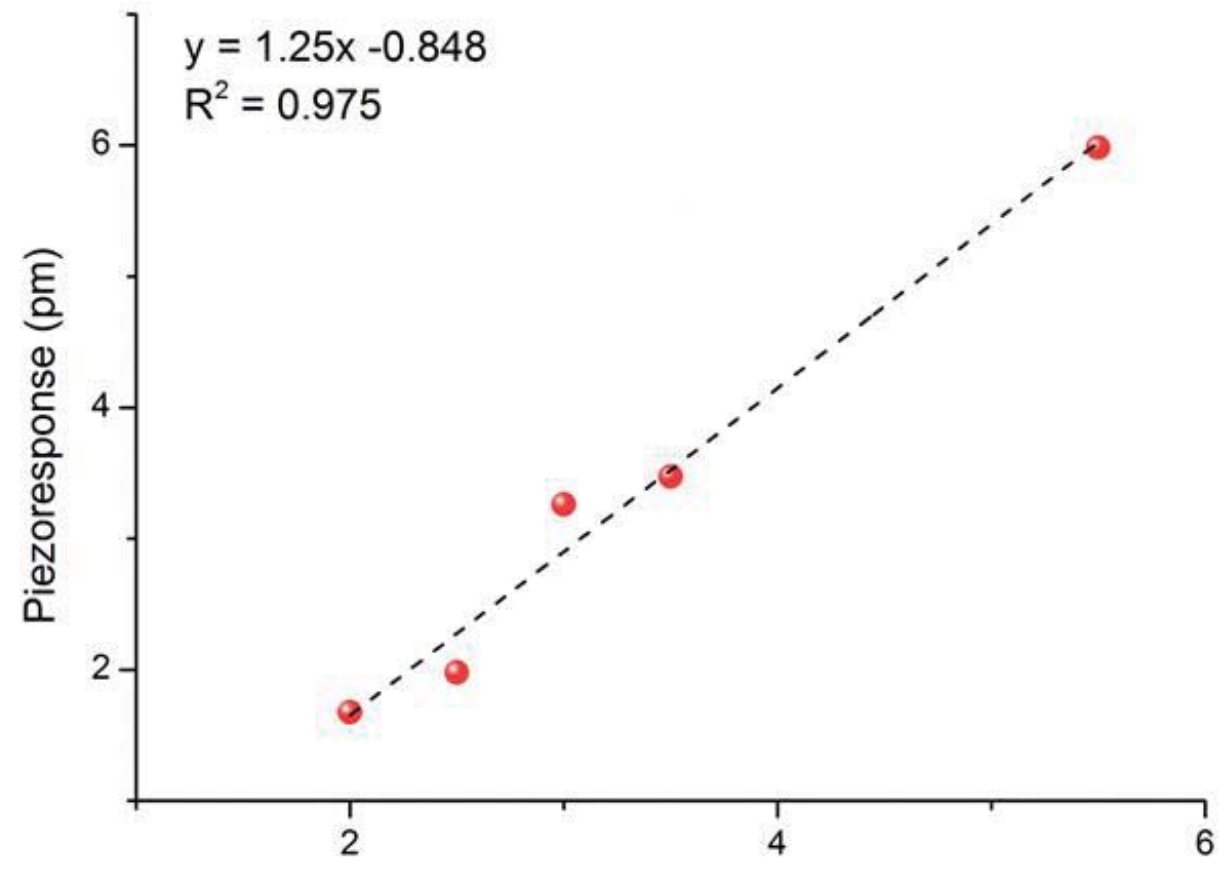

Drive Amplitude $\left(\mathrm{V}_{\mathrm{ac}}\right)$

Figure S4. Piezoresponse as measured by vertical DART-PFM switching spectroscopy as a function of drive voltage (2.0 to $5.5 \mathrm{~V}$ ) at a constant DC bias of $15 \mathrm{~V}$. Note that the piezoresponse has not been normalized to $\mathrm{pmV}^{-1}$, it has been displayed in picometers $(\mathrm{pm})$ to allow for the change in piezoresponse as a function of voltage to be demonstrated. 
PFM Response Modelling

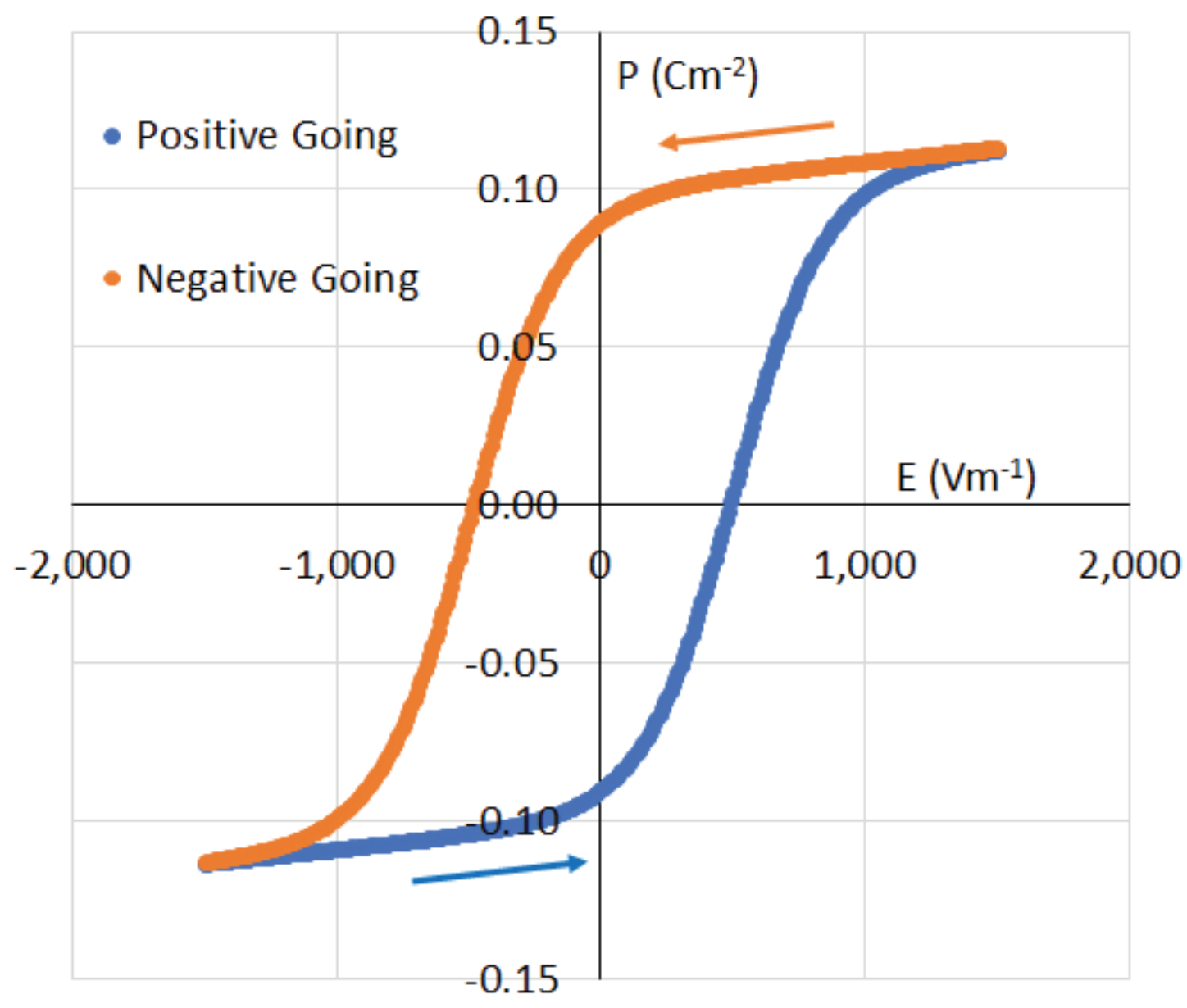

Figure S5. Model of ferroelectric hysteresis based on the function shown in Equation (S18) and (S19). $\left(P_{S}=0.1 \mathrm{Cm}^{-2}, P_{R}=0.09 \mathrm{Cm}^{-2}, E_{c}=500 \mathrm{Vm}^{-1}, \varepsilon_{\text {opt }}=2\right)$. 